\title{
Group formation: The interaction of increasing returns and preferences' diversity $^{1}$
}

Gabrielle DEMANGE ${ }^{2}$

July 13, 2004

\footnotetext{
${ }^{1}$ This is written for the volume Group formation in economics: networks, clubs, and coalitions edited by Gabrielle Demange and Myrna Wooders. This chapter is not intended to be a survey, and is quite naturally influenced by my own work.

${ }^{2}$ EHESS-DELTA, CEPR, address : DELTA 48 bd Jourdan, 75014 Paris, e-mail demange@delta.ens.fr. I thank Francis Bloch and Myrna Wooders for helpful remarks.
} 


\section{Contents}

1 Introduction 3

2 Competition under increasing returns 5

2.1 An economy with differentiated goods . . . . . . . . . . . 6

2.2 Sustainable oligopoly configuration . . . . . . . . . . . . 8

2.3 Existence of a sustainable oligopoly configuration . . . . . . . . . 10

2.3.1 Some simple cases . . . . . . . . . . . . . . . . 10

2.3.2 Intermediate preferences . . . . . . . . . . . . . . 12

2.3 .3 Existence result . . . . . . . . . . . . . . . . 13

2.4 On the optimality of a sustainable configuration . . . . . . 15

2.5 Related framework . . . . . . . . . . . . . . . 16

$\begin{array}{lll}3 & \text { On competition across groups } & 18\end{array}$

3.1 Games without spillovers . . . . . . . . . . . . . . 18

3.2 Incentives to form coalitions . . . . . . . . . . . . . 20

3.2 .1 Increasing returns to coalitions . . . . . . . . . . 20

3.2.2 Superadditivity, coalition structures and efficiency . . . . 21

3.3 Free mobility . . . . . . . . . . . . . . . . . 23

3.4 Free entry coalition structures . . . . . . . . . . . 25

3.5 Free mobility, free entry, and increasing returns . . . . . . . 26

3.6 Restrictions on free entry or on preferences . . . . . . . . . 27

4 Public decision rules and mobility 30

4.1 Fiscal tools, decision rules and increasing returns . . . . . . . . 30

4.2 Blocking under negative externality . . . . . . . . . . 33

4.3 Free mobility with a fixed number of jurisdictions . . . . . . . 34

5 Conclusion $\quad 37$ 


\section{Introduction}

In a large range of political and economic situations a group of individuals sharing common interests can pursue them more efficiently through a coordinated action. Returns to coordinated action explain why decisions are conducted within organized groups. Most often also, individuals differ in some aspects. The diversity in individual tastes hampers a full exploitation of coordination, and encourages the splitting of society into smaller self sufficient groups. The main purpose of this chapter is to analyze group formation under these two opposite forces : increasing returns to size and to coordination on the one hand, and heterogeneity of preferences on the other. In particular, we try to understand which kind of competition among groups allows for an efficient and stable organization. Competition among firms under increasing returns to scale and competition among large communities are two prominent domains of application.

Most often there is a tension between the exploitation of the potential gains to collective action and the introduction of some form of competition among groups. To illustrate this tension, consider an industry in which the exploitation of increasing returns to scale calls for a small number of active firms, each one serving a large set of customers. As a result, competition between active firms is softened. The proponents of contestable markets argue that the perfect freedom of entry and exit in the industry introduces a very strong competitive force, which promotes efficiency (Baumol, Panzar, and Willig (1982)). This force however is so strong that it may result in instability, which is a situation sometimes referred to as "destructive" competition.

A similar difficulty arises more generally when the formation of groups is subject to two "natural" competitive pressures generated by free mobility and free entry ${ }^{3}$. Under free mobility, each individual can choose any existing group without restriction. Under free entry any new group can form and coordinate action without cost. The difficulty is that on one hand free mobility equilibria exist but are typically numerous and inefficient, and that on the other hand a free mobility and free entry equilibrium is efficient but may fail to exist.

At this point, it is useful to qualify this claim and to divide the situations of group formation crudely into two categories. In the first category, almost all gains to cooperation are realized through small coalitions. Then, the competitive pressure exercised by individuals (free mobility) is powerful enough to ensure near optimality ${ }^{4}$, as made precise by Chapters 6 and 7 in this volume. This chapter instead analyzes the second category of situations in which, owing to increasing returns, efficiency and competition across groups are likely to foster the formation of large coalitions. One of our main objectives is to identify clear conditions under which free mobility and free entry are compatible in that

\footnotetext{
${ }^{3}$ See for example the analysis of competition among jurisdictions by Bewley (1981).

${ }^{4}$ The intuition was given by Tiebout (1956) in what he called the severe model: "Let the number of communities be infinite and let each announce a different pattern of expenditures on public goods. [...] The consumer-voters will move to that community which exactly satisfies their preferences." p. 421.
} 
set $\mathrm{up}^{5}$.

Competition among firms under increasing returns to scale is first investigated. Firms select the characteristics of their products, and individuals differ in their tastes or income. The technology exhibits increasing reruns to scale. The main issue is whether free entry is bound to be destructive, calling for some regulation. Although there are increasing returns to scale, differences in individuals may allow for several active firms, each one attracting a different group of customers through an appropriate product design and price policy. Thus, the idea of contestable markets must be extended to an oligopoly. If entry is free any opportunity to make a positive profit with an alternative offer targeted towards a group of customers triggers entry. Under some conditions, free entry competition is not destructive, but instead results in a sustainable oligopoly configuration in which a stable and optimal variety of products is offered (Demange and Henriet (1991)). One condition bears on preferences, and requires a specific form of heterogeneity called intermediate preferences. The other crucial condition is to allow the number of active firms and their offers to be endogenous, affected by the relative strength between the increasing returns to scale and the individual tastes heterogeneity.

To what extent is competition across groups in other settings similar to competition among firms ? Consider, for example, jurisdictions. Choosing public goods levels and their financing is subject to objections stemming from part of the population. Also these decisions are affected by the ability of individuals to move to another jurisdiction or to another country. Accordingly, free entry and free mobility are competitive forces at work. Also public goods generate returns to scale. If jurisdictions are viewed as controlled by land owners/developers who behave as firm managers, competition among jurisdictions is similar on many aspects to competition among firms under increasing returns to scale. Jurisdictions however are run by elected people, and the tax tools that can be used may be limited. How do these features affect competition across jurisdictions? Similarly, issues such as stratification, fiscal competition, break-up of nations, can be discussed within a model of competition among regions, or countries ${ }^{6}$. If one is willing to highlight such issues, the robustness of the results to the specific modeling assumptions should matter.

Cooperative game theory provides useful tools for conducting such a robustness analysis. Indeed, free entry, which encompasses the possibility for a new group to form if it is in the interest of its members, is basically a no-blocking condition. In the situations we are interested in, the splitting of the society into smaller self-sufficient groups may be an efficient outcome : the underlying game is not necessarily superadditive contrary to the standard set up ${ }^{7}$. It turns out that, under a condition of no spillovers across coalitions, standard results

\footnotetext{
${ }^{5}$ See also chapter 8 in this volume for a complementary analysis.

${ }^{6}$ Following Westhoff (1977) the literature on these issues is large. See Guesnerie and Oddou (1981), Greenberg and Weber (1986), and more recently Alesina and Spolaore (1997) to name a few.

${ }^{7}$ Superadditivity may fail even under "technological" increasing returns to scale. This failure is due to the very fact that individuals differ and that, most often, personalized prices or personalized taxes cannot be charged.
} 
on the core are very useful. These results shed some light on conditions that are somewhat necessary for a stable and efficient partition of the society into self-sufficient groups to result from free entry and free mobility.

Albeit useful, the core approach is not appropriate in all situations. In particular, the interaction between free mobility and free entry is properly addressed by the core only if returns to coalitions are increasing. Returns to coalitions fail to be increasing in situations in which an individual who wants to join a group imposes a negative externality on some members of that group. This failure can be due to various factors. For instance constitutional constraints, such as the use of majority rule within coalitions, may prevent all members from benefiting from a newcomer even if a Pareto improvement would be feasible. Most importantly, adverse selection generates negative externalities. In such a context, if a group is unable to screen newcomers, the ability of individuals to move places a major constraint on the decisions within groups ${ }^{8}$. Jurisdictions that perform redistribution among citizens typically face such constraints, as shown for example by Epple and Romer (1991). Therefore addressing competition among groups under negative externalities is an important, although difficult, topic. This chapter concludes by discussing this issue in a simple public goods economy.

The chapter is organized as follows. Section 2 focuses on competition in a simple economy under increasing returns to scale and heterogeneous consumers. The concept of sustainable oligopoly is discussed and analyzed. Section 3 studies in a more general and abstract set up competition among groups in the absence of spillovers. Whereas Section 3 develops some insights of Section 2, it can be read first. Finally Section 4 analyzes public decisions in a simple public good economy through the previous approach, and addresses the interaction between free mobility and free entry under negative externalities.

\section{Competition under increasing returns}

Most often, whereas economic activities are performed under some form of increasing returns to scale, exploiting these returns imposes constraints on consumers. For instance, a unique large shopping center instead of several smaller ones may save on managerial cost but requires a longer travel time for some customers. Also exploiting increasing returns to scale in the production of a good, a cultural good for instance, entails standardization and uniformity. The greater the variance across individuals' tastes, the more likely it is that some individuals will be dissatisfied. As a result, a group of similar customers, who are close in their location or in their tastes or in their income, may be better off by being offered a service closer to their aspirations, even if the lower scale of production entails a larger price.

The purpose of this section is to explore how competition works in a simple economy that combines these two features : increasing returns to scale, which

\footnotetext{
${ }^{8}$ In competitive insurance markets, the constraints stemming from adverse selection have been well known since the work of Rothschild and Stiglitz (1976).
} 
promotes standardization, and the individuals' preferences diversity, which promotes differentiation ${ }^{9}$. We first describe the economy, and give some examples.

\subsection{An economy with differentiated goods}

We consider an economy with two goods: money and a differentiated consumption good. The consumption good may have different characteristics such as location, quality, color. It can be private or public, divisible or indivisible, or be associated with a package of products such as the access through a network to various services.

- Offers

An offer specifies the parameters of the transaction proposed by a producer. For a divisible good by example, an offer is represented by a vector $\left(q_{1}, q_{2}, p, t\right)$ where $q_{1}$ is the quality of the product, $q_{2}$ is its location, $p$ is its unit price, and $t$ is a fixed fee. If the good is public, it is simply $\left(q_{1}, q_{2}, t\right)$ where $q_{1}$ and $q_{2}$ are interpreted as above and $t$ may be either a tax fee (poll tax) or a proportional scheme based on observed income. The set of a priori possible offers is a subset $A$ of a finite dimensional space, which may be discrete in case of indivisible goods.

\section{- Consumers}

The set of consumers is finite, denoted by $N$. Each individual is interested in one offer at most. Thus preferences are defined on the set $A: u_{i}(a)$ denotes the utility level that consumer $i$ achieves by choosing offer $a$. For instance, if the good is divisible, this level represents the indirect utility obtained by choosing the optimal quantity. Consumers also have an outside option, say of buying no good and paying nothing. This option is represented by a fictitious offer in $A$ denoted by $a_{0}$.

\section{- Production}

The number of firms is not given a priori. The same technology is available to each potential firm. The profit of providing an offer $a$ to a set $S$ of consumers is denoted by $\pi(a, S)$. By convention, the profit associated with the fictitious offer is null whatever set $S: \pi\left(a_{0}, S\right)=0$. As said in the introduction, we are interested in situations where production is performed under some form of increasing returns to scale.

There are increasing returns to scale if $\pi(a, S) \geq 0$ implies $\pi\left(a, S^{\prime}\right) \geq 0$ for any $S^{\prime}$ containing $S$.

This condition states a weak form of increasing returns to scale: any offer that makes no loss when chosen by a set of customers makes no loss either if chosen by more customers. For instance let offer $a=(q, t)$ represent the access to a service with quality $q$ at a fixed fee $t$. Returns to scale are increasing if, given quality $q$, the cost of production is composed of a fixed cost $f(q)$ plus a variable cost $s c(q)$ proportional to the number of customers $s$. A null marginal cost, $c(q)=0$, represents the case of a pure public good.

\footnotetext{
${ }^{9}$ This section mainly builds on Demange and Henriet (1991).
} 
Because each firm has access to the same technology, established firms do not enjoy cost or reputation advantages. Accordingly our approach takes a "long run" perspective, in which all costs, including research and development, determine the profit function.

Examples. Product differentiation, either vertical or horizontal, price discrimination, and congestion effects can be analyzed in the preceding framework.

- Example 1. Differentiation models have been extensively used to study imperfect competition.

In horizontal differentiation models à la Hotelling (1929), individuals differ in their "location". Each individual is characterized by his location parameter, $\theta$. The parameter can represent a true location or, more generally, be interpreted as a characteristic specifying for example preferences over colors, or over movies (see the survey of Gabszewicz and Thisse (1986)). Most often, the parameter is assumed to belong to a line, or to a circle. An offer $a=(q, p)$ specifies the location of the product and its unit price. Each individual buys zero or one unit of a product, a carfor example, and derives a utility which depends on the "distance" between his location and that of the product, as given for example by

$$
u(q, p, \theta)=v-p-f(|q-\theta|)
$$

where $f$ is a convex transportation cost. Competition encourages firms to differentiate according to their location, thereby attracting groups of consumers that are located in non-overlapping zones.

Vertical differentiation models are similar except that products differ in quality instead of location. All individuals agree on the ranking of quality but differ in their valuation for it, say because they differ in their income level $\theta$ (Shaked and Sutton (1993)). An offer $a=(q, p)$ specifies the quality of the product and its unit price. Examples of utility functions are $u(q, p, \theta)=q(\theta-p)$ or

$$
u(q, p, \theta)=v(q, \theta)-p
$$

under a Spence-Mirless condition $\frac{\partial^{2} v}{\partial \theta \partial q}>0$ which says that the marginal utility for quality increases with the characteristic $\theta$. Here competition should lead firms to differentiate according to the quality of the product they offer, thereby attracting groups of individuals with different income levels or different valuations.

- Example 2. Price discrimination Consider an homogeneous good, without scope for differentiation either through quality or location. Firms may nevertheless discriminate between customers by proposing two-part tariffs. Thus an offer is written as $a=(t, p)$ where $t$ is the access cost, and $p$ is the unit price. Given a utility function $v(x, \theta)+m$ where $m$ is the numeraire and $x$ is the amount of the good consumed (number of visits, telephone calls and so on), an individual facing tariff $(p, t)$ chooses the optimal quantity, and compares different offers according to the indirect utility

$$
u(p, t, \theta)=\max _{x}\{v(x, \theta)-p x-t\} .
$$


- Example 3. Communications network The characteristics of a product represent all the services to which an individual may have access. They can be represented by the set of connections between two points: $q=\left(q_{i, j}\right)$ in which $q_{i, j}$ is equal to 1 if $i$ is joined to $j$ through the network and 0 otherwise. Whenever the cost to serve a set of customers through a given network consists of the sum of a fixed cost of building the network, $f(q)$, and a cost proportional to the number of users, increasing returns to scale are met ${ }^{10}$.

- Example 4. Congestion Let an offer represent the access to a service, the quality of which is endogenous, affected by the number of customers who have access to the service. A minimal capacity is needed to provide the quality $q$ to a number of users $s$, denoted by $K(q, s)$. There are congestion effects if the capacity needed to ensure a given quality increases as the set of consumers expands: $K(q, s)$ increases with $s$. Profit is given by

$$
\pi(q, t, S)=s t-C(K(q, s))
$$

where $s$ is the number of individuals in $S$ and $C(k)$ is the cost of building capacity $k$. Increasing returns to scale hold if the per user cost does not increase with the number of users, that is if $C(K(q, s)) / s$ does not increase with $s$. So congestion effects and increasing returns to scale are compatible: both are present if the cost to provide a given quality to a number of users increases with the number of users (congestion) but less than proportionately to that number (increasing returns). This form of congestion is not too severe, weaker than in club theory, as explained below.

These examples provide an idea of the trade-off faced by firms. On one hand, due to the differences in individuals' tastes, firms have incentives to differentiate by offering products with different characteristics so as to avoid sharp competition. On the other hand, exploiting increasing returns calls for products that attract as many consumers as possible. We now turn to the analysis of this trade-off in a competitive framework.

\subsection{Sustainable oligopoly configuration}

Competition is analyzed here through the equilibrium concept of sustainable oligopoly. Other approaches and related framework are discussed at the end of this section.

Before defining sustainability, we need to describe how an oligopoly may organize itself. An oligopoly configuration specifies for each active firm its proposed offer and its (nonempty) set of customers, hereafter $\left(a_{\ell}, S_{\ell}\right)$ for firm $\ell$. It

\footnotetext{
${ }^{10}$ Increasing returns to scale differ from the property of economies of scope. Economies of scope prevail if the cost of connecting two nodes decreases as the extending network expands. Therefore the property specifies how the fixed cost $f$ varies with the network $q$ (see Sharkey (1982) for an analysis of competition in this setup). More generally, economies of scope can be defined as follows in our model. Consider two firms, each one proposing an offer, say $a$ and $a^{\prime}$, to two disjoint sets of customers $S$ and $S^{\prime}$. There are economies of scope if the sum of their cost is larger than the cost borne by a unique firm that jointly proposes these two offers to the same sets of customers. We preclude this form of economies of scope. Note, however, that economies of scope are possible within the different components of a multi-dimensional offer $a$.
} 
is important to allow the number of firms/offers to vary: this number, denoted by $L$, must be affected by the strength of the increasing returns to scale relative to the scope of preferences' heterogeneity. Also, recall that customers have the option of buying nothing (option denoted by the fictitious offer $a_{0}$ ). Let $S_{0}$ denote the (possibly empty) set of customers who choose that option. It is convenient to include $\left(a_{0}, S_{0}\right)$ in a configuration, even if $S_{0}$ is empty. Because each individual is interested in one offer at most, the various groups of customers form a partition of the whole set $N$.

Definition 1 An oligopoly configuration is given by a set of pairs $\left(a_{\ell}, S_{\ell}\right)_{\ell=0, \ldots, L}$ in which $S_{\ell}$ is the set of customers of offer $a_{\ell}, S_{\ell}$ is nonempty for $\ell>0$, and $\left(S_{\ell}\right)_{\ell=0, \ldots, L}$ is a partition of the set of consumers $N$. The configuration is feasible if $\pi\left(a_{\ell}, S_{\ell}\right) \geq 0$ for each $\ell=1, \ldots, L$.

In the sequel, given a partition of $N, \ell(i)$ denotes the set to which $i$ belongs.

Our purpose is to explore how free competition between all firms, active and potential, affects an oligopoly configuration. Sustainability basically describes an equilibrium in a competitive environment in which each consumer can choose whatever offer is proposed, and each firm, active or not, has the opportunity to propose a new offer.

Definition 2 A configuration $\left(a_{\ell}, S_{\ell}\right)_{\ell=0, \ldots, L}$ is sustainable if (free choice/free mobility) each consumer chooses among the offers he prefers:

$$
\text { for each } i, u_{i}\left(a_{\ell(i)}\right) \geq u_{i}\left(a_{k}\right) \text { for each } k=0, \ldots, L
$$

(firms make no loss) $\pi\left(a_{\ell}, S_{\ell}\right) \geq 0$ for each $\ell=1, \ldots, L$

(free entry) there are no offer $b$ and subset of customers $T$ that satisfy

$$
\pi(b, T) \geq 0 \text { and } u_{i}(b)>u_{i}\left(a_{\ell(i)}\right) \text { for each } i \in T .
$$

Free choice/free mobility states the standard behavior of consumers who are "product and price" takers. This behavior can be justified in an infinite population, as we will see in section 2.3.3. The no loss condition is clear. Free entry says that no firm, active or not, has the opportunity to propose a new offer that would attract some consumers and make a profit. This condition needs more comment.

It should first be noted that free entry is a strong competitive force. To see this, consider the situation where an offer $(q, p)$ specifies the quality of a product $q$ and the unit price $p$. Assume that the price $p$ can be changed by any small amount. Free entry implies competition in prices à la Bertrand, which drives profit down to zero. The argument is standard, based on an undercutting strategy: if firm $\ell$ makes positive profit, an entrant can propose a product with an identical quality $q_{\ell}$ but at a slightly lower unit price than $p_{\ell}$. All customers in $S_{\ell}$ are better off while the entrant makes a positive profit, in contradiction to free entry. By a similar argument each firm maximizes profit since otherwise entry would be profitable. More generally, profits are driven to zero under free entry whenever at least one characteristic in the offer -price or quality or 
location- can be adjusted in a continuous way. Thus, free entry is indeed a very competitive force. We now discuss two factors that might raise difficulties for this force to be effective.

First, the free entry condition makes sense if any firm, established or potential, faces the same profit function. It may be argued that fixed costs in production generate an asymmetry. Even worse, the fact that established firms have already incurred the fixed costs would create an advantage in their favor vis à vis potential entrants: fixed costs would constitute barriers to entry. As argued by Baumol et al., established firms do not enjoy any advantage if fixed costs are recoverable, which means that firms recover without a loss the fixed costs they may have incurred during their activity when they leave the market. Then any possibility of making profit triggers entry, possibly for a short time, since exit is free as well (the so-called hit and run strategy): this justifies the free entry condition ${ }^{11}$.

Second, the threat of entry underlies the free entry condition. The threat is credible only if firms have enough information on customers. A firm, incumbent or entrant, must know that there is an offer preferred by a group of customers to their current choice and that selling that offer to that group will be profitable. This informational requirement is rather weak under increasing returns to scale. To see this note that whenever entry is profitable with an offer attracting a given set of customers, it is also profitable by attracting even more customers. Therefore an entrant does not need to screen among the consumers, and can agree to serve any consumer who asks to be served. Accordingly to decide whether entry is profitable, a firm only needs to know the distribution of characteristics or tastes within the population.

\subsection{Existence of a sustainable oligopoly configuration}

As we have just seen, sustainability imposes demanding requirements. A first task is to examine whether a sustainable configuration exists under reasonable conditions. Simple examples first illustrate the problem.

\subsubsection{Some simple cases}

Increasing returns and preferences heterogeneity generate two opposite forces. In the two first examples below, one of the two forces does not operate. In such situations, a sustainable configuration exists, which moreover displays a very simple structure. In the third example, the two opposite forces operate, leading to a trade-off that raises existence problems.

Increasing returns to scale and homogeneous population. Intuitively, if individuals are all alike and there are increasing returns to scale, there is no rationale behind a variety of different offers. This is indeed true.

\footnotetext{
${ }^{11}$ If fixed costs are (partially) sunk, i.e. cannot be entirely recovered, then indeed an asymmetry between active firms and potential entrants arises : an incumbent, who has already paid the sunk costs and knows that an entrant has not, may have a credible incentive to react to entry. For a discussion on these issues see Gabszewicz and Thisse (2002).
} 
Consider configurations of the form $(a, N)$ in which a unique offer is bought by the whole set of consumers. Choose an "efficient" offer $a^{*}$, which maximizes each (identical) consumer's utility, $u(a)$, under the no loss condition $\pi(a, N) \geq 0$ (most often $a^{*}$ is unique). The configuration $\left(a^{*}, N\right)$ is sustainable : any other offer that is strictly preferred by a group of customers will surely result in a loss. Furthermore, all sustainable configurations are obtained that way under mild additional assumptions ${ }^{12}$. As an illustration, consider example 4, where a public service is subject to some mild form of congestion. Sustainability requires all customers to access the same service, the quality of which maximizes each individual's utility under average cost pricing, i.e. quality $q$ that maximizes $u(q, C(K(q, n)) / n)$.

Decreasing returns to scale. Returns to scale are (weakly) decreasing if any offer that makes no loss when chosen by a set of customers makes no loss either if chosen by fewer customers. Consider the "autarky" configuration in which each consumer produces his preferred good for his own consumption under the no loss condition (so there are as many offers as consumers). If there are decreasing returns to scale, autarky is clearly sustainable ${ }^{13}$.

As these two extreme cases make clear, it is the exploitation of increasing returns to scale by firms facing different types of individuals that generates a non trivial variety of distinct offers, each one attracting a group of possibly different types of customers.

Destructive competition. We define an economy characterized by three parameters: the individuals' valuation for a public good, say a swimming pool, the cost of producing one unit of the public good, and a transportation cost. The example illustrates the two following points:

1. the number of offers at a sustainable configuration (if any) depends on the economy parameters,

2. a sustainable configuration fails to exist for a non-negligible set of parameter values.

Three towns, $X, Y, Z$, each populated with the same number of citizens $s$, are equidistant along a circle at a distance $d$ from each other. An offer specifies the location of a swimming pool and the access price to use it, common to all individuals. Citizens are all alike, except for their location. Their valuation for the swimming pool is $v$, and the per unit transportation cost is $c$. Thus, the utility of an individual who pays $p$ for the access to a swimming pool at a

\footnotetext{
${ }^{12}$ Consider a sustainable configuration. If a unique offer $a$ is proposed, it satisfies $\pi(a, N) \geq$ 0 ; $a$ must be efficient, since otherwise an efficient offer would attract all consumers and be profitable. If distinct offers are proposed, free mobility imposes each individual to be indifferent between all the proposed offers. Let offer $a$ be bought by $S$, a strict subset $N$. If increasing returns to scale are strict, $0 \leq \pi(a, S)<\pi(a, N)$. So if offers can be adjusted slightly, for example if the price component is in small enough units, a new offer can be designed so as to make each consumer strictly better off and generate profits.

${ }^{13}$ Assuming the number and the characteristics of offers to be endogenous is crucial to get the sustainability of autarky. Under constraints on the number of firms for instance, autarky might not be feasible.
} 
distance $l$ is

$$
v-p-c l .
$$

The once for all cost of building a swimming pool is $F$. So $f=F / s$ denote the per citizen cost if a swimming is used by the citizens of only one town.

We claim that no sustainable configuration exists if

$$
v<f,(f+c d) / 2<v \text { and } v<f / 3+c d .
$$

Consider a sustainable configuration.

First, if $v<f$, there is at most one swimming pool. To show this, note that a swimming pool is necessarily used by more than one third of the population (otherwise the access price would be larger than $f$ and no citizen would use the swimming pool). This excludes three swimming pools. This also implies that in the case of two swimming pools, the citizens of one town, say $X$, use both. By free mobility, the $X$-inhabitants are indifferent between the two places. Lowering the access price to one swimming pool slightly without changing its location, would attract all the $X$-inhabitants, thereby strictly increasing profits : sustainability requires a unique swimming pool.

Second, if $(f+c d) / 2<v$, there is surely one swimming pool : otherwise a swimming pool located equidistant between two towns at an access price slightly larger than $f / 2$ would make all the citizens of the two towns better off and generate profits : entry would be profitable.

Third, if $v<f / 3+c d$, citizens at a distance larger than $d$ from a swimming pool prefer not to use it, even if they pay $f / 3$, the smallest possible access price. Thus under (2), we are left with only one possibility: a configuration with a unique swimming pool, located say between $X$ and $Y$, possibly closer to $X$, and used by $X$ and $Y$ only. The configuration is not sustainable: entry between $Z$ and $Y$, a little closer to $Y$ than $Z$, at an access price slightly larger than $f / 2$, attracts all citizens of $Y$ and $Z$ and makes profit.

This last example shows that a sustainable configuration may not exist even under standard preferences. It should be clear that if the towns were located along a line instead of a circle symmetry would be broken, and a sustainable configuration would exist. It turns out that existence in such a case follows from a more general existence result (theorem 1 below). Before stating this result, we need to define the intermediate preferences property, which was introduced in social sciences by Kemeny and Snell (1962) (see also Grandmont (1978)).

\subsubsection{Intermediate preferences}

The property bears on a family of preferences, all defined on the same set. Here we restrict attention to preferences that are parameterized by a one dimensional characteristic. It turns out that this restriction is satisfied in the standard models of product differentiation and price discrimination described in section 2.1.

Consider orders of preferences on $A$. An order is said to be between two other orders if it ranks alternative $a$ before $b$ whenever the two others both rank $a$ before $b$. Consider now a family of preferences that are parameterized by a 
one-dimensional parameter. Given three parameters, one parameter is between the other two. The property of intermediate preferences holds if the associated preferences follow the same comparison ${ }^{14}$. Formally:

Definition 3 The family $\{u(., \theta), \theta \in[\underline{\theta}, \bar{\theta}]\}$ of utility functions on $A$ indexed by the one dimensional parameter $\theta$ defines intermediate preferences if, for any a and $b$ in $A$, the sets $\{\theta, u(a, \theta)>u(b, \theta)\}$ and $\{\theta, u(a, \theta) \geq u(b, \theta)\}$ are intervals.

So the property specifies that the preferences in the family are related among each other while preserving some heterogeneity in tastes. Whereas the characteristic is a scalar, alternatives may be multi-dimensional. Consider some examples of section 2.1.

In a vertical differentiation model where $u(q, p, \theta)=v(q, \theta)-p$, the SpenceMirlees condition, $\frac{\partial^{2} v}{\partial \theta \partial q}$ of constant sign, ensures that preferences are intermediate ${ }^{15}$. In a horizontal differentiation model, consider firms that not only choose their location $q_{2}$ but also the quality of their product $q_{1}$. Utility functions

$$
u\left(q_{1}, q_{2}, p, \theta\right)=v\left(q_{1}\right)-p-f\left(\left|q_{2}-\theta\right|\right)
$$

define intermediate preferences under the standard assumption of an increasing and convex transportation cost $f$. Finally, consider the price discrimination example in which consumers choose the optimal quantity according to a proposed two-part tariff. Indirect utility is given by

$$
u(p, t, \theta)=\max _{x}\{v(x, \theta)-p x-t\} .
$$

A family of intermediate preferences is obtained whenever the utility for the good, $v(x, \theta)$, satisfies the Spence-Mirlees condition (from the first example, it suffices to check that indirect utility $u$ inherits the Spence-Mirlees property).

\subsubsection{Existence result}

We are now ready to state the following existence result.

Theorem 1 Under increasing returns to scale and one-dimensional intermediate preferences, a sustainable configuration exists. Individuals cluster according to their types: if two individuals choose the same offer, all individuals with preferences between them also choose the same offer.

Consider a vertical differentiation model in which offers are of the form $(q, p)$ and individuals preferences given by $u(q, p, \theta)=\theta(q-p)$. The clustering property

\footnotetext{
${ }^{14}$ The definition extends to any set of parameters that is endowed with a "between" relation, such as a convex set. Intermediate preferences hold if given three parameters where one parameter is between the other two, then the associated preferences follow the same comparison. Contrary to a one dimensional parameter, three parameters may not be comparable, in which case the corresponding preferences are not necessarily comparable either.

${ }^{15}$ Given $a=(q, p)$ and $a^{\prime}=\left(q^{\prime}, p^{\prime}\right)$, let $I=\left\{\theta / v(q, \theta)-v\left(q^{\prime}, \theta\right)>p-p^{\prime}\right\}$. We have to prove that $I$ is an interval. If $q=q^{\prime}, I$ is either empty or the whole interval $[\underline{\theta}, \bar{\theta}]$. If $q \neq q^{\prime}$, the function $\theta \rightarrow v(q, \theta)-v\left(q^{\prime}, \theta\right)$ is strictly monotone thanks to the Spence-Mirlees condition, hence $I$ is an interval. The proof is similar if the inequality defining $I$ is weak.
} 
implies that all individuals with income $\theta$ within some range choose the same offer. By free mobility, the quality of the product chosen by individuals is increasing in their income. More generally, the clustering property means that each offer is bought by all individuals whose characteristic belongs to a given interval of characteristics, hereafter called "interval" of individuals.

The proof is constructive (see Demange and Henriet (1991) for details). One first builds a configuration that satisfies two adequate properties ${ }^{16}$ :

(a) $S_{\ell}$, the set of consumers who buy a given offer $a_{\ell}$, is an interval

(b) no firm can enter profitably by attracting an interval of customers, that is free entry holds for "intervals" of customers.

Thanks to intermediate preferences, a single-peaked preferences property is satisfied at the configuration: the utility of an individual decreases over the offers chosen by individuals with either increasing characteristics, or decreasing ones. Using increasing returns to scale, this property implies that the free entry condition holds whatever group of customers.

Discussion Intermediate preferences can be defined for parameters that are not necessarily one dimensional (see footnote 14). A natural question is whether Theorem 1 extends to these settings. We consider two cases.

First let characteristic $\theta$ belong to a multi-dimensional convex set. Preferences are intermediate if the sets $\{\theta, u(a, \theta)>u(b, \theta)\}$ and $\{\theta, u(a, \theta) \geq u(b, \theta)\}$ are convex. Unfortunately Theorem 1 does not extend to that setting, that is a sustainable configuration does not necessarily exist if intermediate preferences and increasing returns hold but the characteristic is multidimensional (see Demange and Henriet for an example).

Second, let the characteristic belong to a graph ${ }^{17}$. Preferences are intermediate if the two sets $\{\theta, u(a, \theta)>u(b, \theta)\}$ and $\{\theta, u(a, \theta) \geq u(b, \theta)\}$ are connected. The existence of a sustainable configuration is ensured if the graph is a tree (see section 3.6), but not if the graph contains a cycle as shown by the example in section 2.3.1.

Finally Theorem 1 holds if, instead of the intermediate preferences assumption, preferences are assumed to be single peaked on a one-dimensional set of alternatives (Greenberg and Weber (1993)). While these preferences assumptions $^{18}$ differ, they both entail a one-dimensional requirement, either on

\footnotetext{
${ }^{16}$ Order individuals by their characteristics. Starting with individual $n$, define "guarantee levels" inductively as follows: $\gamma(n)$ is the maximum utility level that individual $n$ can get by himself; $\gamma(i)$ is the maximum utility level that $i$ can get over offers that are feasible if bought by an interval of consumers $[i, i+r], r$ nonnegative, and give to each $j$ in that interval his guarantee level $\gamma(j)$ at least. There is a configuration that gives to each individual his guarantee level at least is obtained: first by construction there is a coalition say $\left[1, i_{1}\right]$ that can ensure to each of its members the level $\gamma(i)$; second, if $i_{1}<n$ there is a coalition, $\left[i_{1}+1, i_{2}\right]$ that can ensure to each of its members the level $\gamma(i)$ and so on. This configuration, conveniently taking care of ties if any, satisfies the two required properties.

${ }^{17}$ Recall that a graph or a network $g$ on $N$ is a set of unordered pairs, or links, of distinct elements of $N$. A path of $g$ is a sequence $i_{1} \cdots, i_{m}$ where $\left(i_{k}, i_{k+1}\right)$ are links for $k=1, \ldots, m-1$ and are all distinct. A graph is a tree if any two distinct elements are linked by a unique path. A subset $S$ of $N$ is $g$-connected if the path between two elements of $S$ is contained in $S$ (see also the chapters in the first part of this volume).

${ }^{18}$ Two preferences with the same peak do not necessarily coincide : they can differ on the
} 
the set of alternatives (for single peaked preferences) or on the set of characteristics (for intermediate preferences). Section 3.6 below investigates the natural question of whether this unidimensionality requirement can be dropped.

Justifying competitive customers According to the free choice/free mobility condition, individuals are "price and product takers". Such a competitive behavior is justified if each individual has a negligible impact on the product and the price that is chosen by a firm. This occurs if there are many consumers per offer. The question is whether a sustainable oligopoly exists with this property. The natural setup to investigate this question is a continuum of consumers. Assume that an offer is not profitable if a small proportion of the population buys it. More precisely, normalizing the size of the whole population to 1 , there is a size $s>0$ such that whatever offer, it makes a loss if only a group of size smaller $s$ chooses it. Under this additional assumption Theorem 1 extends : a sustainable configuration exists (see Demange and Henriet). Furthermore since each offer must be profitable, each one is bought by an "infinite" number of consumers (at least a proportion $s$ of the whole population), thereby justifying competitive behavior.

Since a sustainable configuration exists under reasonable conditions, efficiency properties can now be investigated.

\subsection{On the optimality of a sustainable configuration}

To assess the optimality of a sustainable configuration, one can check whether all individuals are better off at another feasible configuration. We shall not be limited to that comparison. Feasibility, as defined before, requires each offer to make no loss, which is meaningful in a competitive environment in which each offer is proposed by a different firm. In a context of increasing returns, however, a natural question is whether a monopoly, by proposing several offers and implementing cross subsidies between them, would achieve a Pareto-improvement. If this were the case, an argument in favor of restricting entry and of regulating a monopoly could be made. This leads us to extend the set of feasible configurations by allowing cross subsidies between offers, and to investigate the efficiency of a sustainable configuration within this larger set.

Definition 4 A configuration $\left(a_{\ell}, S_{\ell}\right)_{\ell=0, \ldots, L}$ is feasible, possibly with cross subsidies, if

$$
\sum_{\ell=1, \ldots, L} \pi\left(a_{\ell}, S_{\ell}\right) \geq 0
$$

Equation (3) states the feasibility condition in term of the consumption good, the numeraire. Cross subsidies between offers are allowed because the no loss condition is not required for each offer.

ordering of a pair formed with one alternative to the right and one to the left of the peak. In many examples however, single peaked preferences are parameterized by their peaks and satisfy intermediate preferences. 
Theorem 2 A sustainable configuration is Pareto optimal among all configurations that are feasible possibly with cross subsidies:

if $\left(a_{\ell}, S_{\ell}\right)_{\ell=0, \ldots, L}$ is sustainable and $\left(a_{\ell}^{\prime}, S_{\ell}^{\prime}\right)_{\ell=0, \ldots, L^{\prime}}$ is feasible possibly with cross subsidies it is not true that for each $i u_{i}\left(a_{\ell(i)}\right)<u_{i}\left(a_{\ell^{\prime}(i)}^{\prime}\right)$.

The argument is very simple and general. Let assume by contradiction that a feasible configuration Pareto dominates a sustainable one. By the feasibility condition (3) the profit of at least one offer, say $\left(a_{\ell}^{\prime}, S_{\ell}^{\prime}\right)$, is nonnegative. Since every member of $S_{\ell}^{\prime}$ is better off than under the sustainable configuration, this contradicts free entry.

This result of course implies that a sustainable configuration is optimal among the configurations for which each offer makes no loss. This confirms that free entry, if not destructive, plays a very positive role.

\subsection{Related framework}

Competition under increasing returns or differentiation Two distinct strands in the literature are related to our analysis : the theory of contestable markets, which focuses on increasing returns, and the literature on differentiation, which focuses on the endogenous choice of variants or location.

Baumol, Panzar and Willig consider a "natural monopoly" situation, meaning that a given output vector is produced at a lower cost by one organization than by several. They argue that if the market is contestable, which means that entry is free, a unique firm cannot exploit its monopoly position. The industry is efficiently organized, that is a unique firm is active, without bearing the usual distortions associated with a monopoly. A major difficulty is that an equilibrium under free entry may not exist. The set up considered by Baumol et al. differs from ours in several aspects. Economies of scope in joint production incite firms to propose offers composed with several products, the characteristics of the products are exogenous and the consumers' behavior is described by a demand function. Hence the choice of the characteristics that best suit the customers' taste is left aside, and only the price policy is flexible. In contrast, our modeling of the consumption side allows us to treat firms symmetrically, to know how the demand is split up between several firms, and to determine in an endogenous way the number and the characteristics of the products.

The literature on differentiation uses a different model than here to analyze the choice of products competition. Following Hotelling, a two-stage game is considered in which firms first choose the characteristics of their product (the location on the beach) and second compete in prices. The competition phase may be one shot or repeated, depending on products specifications being somewhat irreversible or not. As usual the existence of an equilibrium and the structure of the economy -the number of competitors and/or products, the efficiency properties- may be quite sensitive to the game. On an elaboration of these issues, see the survey of Gabszewicz and Thisse (1986).

In conclusion, our approach can be viewed as an attempt to integrate the concern of contestable markets into a differentiation model. 
Club theory Club theory basically examines the same question as we do. I outline here only what I see as the main differences. In the initial club model proposed by Buchanan (1965), individuals are identical ${ }^{19}$. The reason why groups form on one hand, and are limited in size on the other hand, is that there is some form of positive externality among individuals but only up to a certain limit in the size of the group: there is a finite "efficient" size for a group. A service subject to congestion is a typical example. Under most equilibrium concepts the population should split into groups all with the efficient size. This is clearly impossible when the overall population size is not a multiple of the efficient size. As a consequence, even in this very simple framework with identical agents, an equilibrium may not exist, due to an "integer" problem. In contrast, such a failure does not exist in our set-up. As seen in section 2.3.1, with identical individuals, a sustainable configuration exists in which all customers choose the same offer : the "efficient" size is always equal to the population size.

Thus the issues we are interested in clearly differ from those of club theory. We are concerned with the difficulties of exploiting increasing returns stemming from the differences in individuals' tastes. Whereas club theory has been extended in various directions, so as to handle heterogeneous population and multiple goods, most developments assume that only "small" groups are effective. In contrast, this chapter considers that large organizations are worth forming even with an infinite population (section 2.3.3).

Jurisdictions and Tiebout equilibrium In the local public goods model proposed by Tiebout (1956), communities set up local taxes and offer various facilities, such as schools, protection, sport centers. Citizens differ in their preferences over local public goods, depending on the number and age of their children, their income level, their leisure activities. So both increasing returns to size and preferences heterogeneity are present. Some political issues such as the location of a government or fiscal decisions (vertical differentiation) under competition between governments can be analyzed by adapting the horizontal and vertical differentiation models introduced in section 2.1. Let us just change our vocabulary as follows:

- a consumer is called a citizen. There is free mobility if each citizen picks the community that best satisfies his preferences.

- a group of consumers is a jurisdiction, or local community,

- an offer specifies the public good characteristics, and how it is financed, possibly through income taxes,

- city managers choose offers ${ }^{20}$ (the location of the good, the level of taxes), and finally the profit is the city budget.

Free mobility simply says that citizens can choose whatever community they wish. Free entry means that no group of citizens could be better off by seceding

\footnotetext{
${ }^{19}$ See the chapters by Kovalenkov and Wooders, and Conley and Smith in this volume for subsequent developments, and the references therein.

${ }^{20}$ It should be clear that this sketched model is a very simplified representation of jurisdictions. In particular it does not address the interaction between city managers and landowners, who could also propose facilities so as to increase the value of land.
} 
and choosing another tax policy and public good levels. In this interpretation a sustainable configuration is called a Tiebout equilibrium. Greenberg and Weber (1986) show that a Tiebout equilibrium exists if jurisdictions are endogenous in an economy in which citizens differ in income only and the public good is financed by either proportional or constant tax scheme. More generally, provided that the assumptions of theorem 1 are satisfied, a Tiebout equilibrium exists. If citizens differ in income only, the clustering property implies stratification.

In this interpretation city managers behave as firm managers. This assumption is somewhat dubious. After all city managers are elected. What is their objective ? The answer to this question is not easy. As is well known, if decisions are to be taken in a democratic way so as to reflect individuals preferences, no objective or decision rule imposes itself. Nevertheless, most often, the way decisions are taken within a group is constrained : decisions must be taken through a specified mechanism ${ }^{21}$. Of particular relevance is the majority rule. To discuss competition across jurisdictions, or more generally across groups, it is convenient to introduce a more general model.

\section{On competition across groups}

Our aim in this section is to highlight the role of some crucial features that shape the formation of groups. In that purpose, we introduce a more general and abstract setting than in the previous section. Three key ingredients must be specified :

- how decisions are taken by the members of a group,

- what are the possible moves for the individuals,

- what are the possibilities for new groups to emerge.

We shall conduct our analysis within the framework of cooperative games. This framework introduces the above specifications in a simple but quite general set up. Given a set of individuals, $N$, sometimes called the society, a non empty subset of $N$ is called a coalition. The primitives are the set of actions (or alternatives, or decisions) that each coalition can take when its members collaborate together, and the associated utility levels or payoffs. In what follows, only games without spillovers will be considered. Although restrictive, the set of situations that can be analyzed through games without spillovers is very rich, starting with competition within jurisdictions. Furthermore the lessons that can be drawn on the formation of groups are in our view important.

\subsection{Games without spillovers}

A game is said to be without spillovers if the actions that are feasible for a coalition if it forms, as well as the payoffs to its members, are not affected by

\footnotetext{
${ }^{21}$ In public good contexts, fixed decision schemes have been considered by many authors starting with Rose-Ackerman (1979), Greenberg and Shitovitz (1988). See also Haeringer (2000), and chapter 8 in this volume, which considers the impact of cost allocation rules on secession in the context of a public project.
} 
the organization of outside agents. In other words, there are no externalities across coalitions as far as feasibility and payoffs are concerned. As will be made clear later on, even in the absence of spillovers, the full organization of the society is relevant to assess its stability. If for instance individuals can move or new groups can form, their opportunities to do so depend on all standing groups $^{22}$.

Without spillovers, the set of feasible actions for a coalition can be described by a single set independently of the organization of outsiders. Let $A(S)$ denote the set of feasible actions for coalition $S$. The welfare of an individual depends on the decision taken by the coalition to which he belongs: If $S$ chooses $a, i$ member of $S$ obtains utility level $u_{i}(a)$. Therefore, each vector of utility levels $\left(u_{i}(a)\right)_{i \in S}$ when $a$ runs in $A(S)$ can be achieved by the coalition to its members. The set of attainable utility levels is assumed to be bounded and closed for each coalition $S$. This is ensured for example if each feasible set $A(S)$ is compact, and utility function $u_{i}$ continuous for each $i$.

The description of the feasible sets depends on the problem at hand. Indeed, the feasible set of a group integrates not only the technological constraints that the group faces if it forms, but also the various "institutional" constraints that may restrict the way decisions are made. For instance, within a community, most often the voting rule is imposed, or the tax tools must be of a specific form, or the information on citizens is poor thereby excluding some types of redistribution. Feasible sets are affected accordingly. In the extreme case where coalitions pick their decision according to a fixed rule, feasible sets boil down to singletons. As a result, the payoff to a player is entirely determined by the coalition to which he belongs : the utility level of individual $i$ who is a member of $S$ can be written simply as $u_{i}(S)$. Aumann and Dreze (1974) introduced these games, and called them hedonic games ${ }^{23}$.

Examples. It should be noted first that this setting encompasses the economy of section 2. Some macro-economic and political issues can also be analyzed though a cooperative game without spillovers.

Example 1. The economy of section 2 is represented by a game without spillovers in which a coalition is a group of customers who set up a firm or a cooperative. A feasible action for $S$ is an offer that makes no loss when each individual in $S$ buys it : $A(S)=\{a, \pi(a, S) \geq 0\}$.

Example 2. The analysis of Jaramillo, Kempf, and Moizeau (2004) also fits our set-up. It provides an explanation for the persistent difference in growth rates across countries by the formation of coalitions of countries. Once a coalition is formed, each country within the coalition decides on its own contribution to a growth enhancing public good, and a hedonic game is obtained. We refer

\footnotetext{
${ }^{22}$ Whereas spillovers (or externalities) across groups, negative or positive, are likely to have an important impact, their analysis is still restricted to specific situations. See for example Yi (1997) for a non cooperative approach to the formation of coalitions, Bloch, chapter 11 of this volume for applications to industrial organization, and Currarini (2003) for an analysis of hierarchical structures.

${ }^{23}$ Hedonic games arise in various non economic contexts, in sport games for example if each player is concerned only with the identity of the team he joins (precluding cash transfers).
} 
the reader to chapter 13 for a precise description and analysis.

Example 3. The formation of nations as analyzed by Alesina and Spolaore (1997) gives rise to a cooperative game without spillovers. Consider a horizontal differentiation model à la Hotelling in which individuals are all alike except for their location. Assuming that individuals are located over an interval, a "country/coalition" is characterized by its borders populated with the individuals living within the interval ${ }^{24}$. A country needs a government, which costs a fixed amount $c$. Within a country, citizens vote on the location of the government using majority rule, and the government cost is financed through an equal tax. Finally assume utility given by the income $y$ minus the tax minus the "preference " distance between the individual's location and that of the government. Under these assumptions, a majority winner exists within a country: the government is located at the median location of the citizens. Therefore a hedonic game is obtained.

For instance, let us compute the payoffs assuming that individuals are uniformly distributed over $[0,1]$. If country $\left[\theta_{1}, \theta_{2}\right]$ forms, the cost per citizen is $c /\left(\theta_{2}-\theta_{1}\right)$, and the government is located at the middle $\left(\theta_{1}+\theta_{2}\right) / 2$. This yields the utility level

$$
y-\frac{c}{\left(\theta_{2}-\theta_{1}\right)}-\left|\theta-\frac{\theta_{1}+\theta_{2}}{2}\right|
$$

to a citizen located at $\theta$ in the country.

Payoffs. Given feasibility sets and utility functions, one can associate the set of payoffs to each coalition ${ }^{25} S$. Here we adopt the so-called Shapley convention, which defines all sets of payoffs as a subset of $\Re^{N}$ (instead of $\Re^{S}$ for $S$ ) as follows

$$
V(S)=\left\{x \in \Re^{N} / \text { there is } a \in A(S) \text { such that } \forall i \in S, x_{i} \leq u_{i}(a)\right\} .
$$

This convention is especially convenient to describe the payoffs that can be achieved by two disjoint coalitions acting separately. Let $S$ and $T$ be two disjoint coalitions. $S$ can achieve any payoff in $V(S)$ to its members and similarly for $T$. Therefore, thanks to the absence of spillovers, any payoff in $V(S) \cap V(T)$ can be achieved by the two disjoint coalitions $S$ and $T$ acting separately.

\subsection{Incentives to form coalitions}

The incentives to form coalitions primarily depend on whether the members of a coalition derive some benefit by accepting new members, whether coalitions gain by splitting into smaller groups. We make these notions precise.

\subsubsection{Increasing returns to coalitions}

We start with notions of increasing returns.

\footnotetext{
${ }^{24}$ If a country is formed with several disconnected intervals, each interval needs to have its own government. This allows one to restrict to intervals.

${ }^{25}$ In the case of transferable utilities, the set of feasible payoffs can be represented more simply by a single number, the "value" to the coalition.
} 


\section{Definition 5}

Feasible sets are increasing if for any two coalitions $S$ and $T$ where $S$ is contained in $T, A(S)$ is contained in $A(T): A(S) \subset A(T)$.

Returns to coalitions are increasing if for any two coalitions $S$ and $T$ where $S$ is contained in $T$, for any a in $A(S)$ there is $b$ in $A(T)$ such that

$$
u_{i}(a) \geq u_{i}(b), \forall i \in S .
$$

Feasible sets are increasing if any feasible action for a coalition remains feasible when new members join that coalition. In the differentiated goods economy of section 2, feasible sets are increasing whenever there are increasing returns to scale. Returns to coalitions are increasing if the sets of payoffs are increasing with coalitions: a coalition can, possibly by changing the current decision, accommodate any newcomer without hurting any of its current member. Clearly increasing feasible sets imply increasing returns to coalitions.

It is important to note that in situations where a fixed decision rule is used, the rule has an impact on whether returns to coalitions are increasing. Indeed increasing returns encompass two conditions. The first is that a coalition can derive gains by accepting newcomers, which is a "technological" increasing returns condition. The second is that the distribution of these gains among the coalition members as induced by the fixed rule makes each one better off. As an example, consider communities that decide on the level and financing of some pure public service so that there are technological increasing returns. If the public good is financed through a poll tax that is chosen according to majority rule, the second condition is not necessarily met. To see this, assume that individuals differ only with respect to their income level. Under standard assumptions, majority rule selects the decision preferred by the voter with median income within the jurisdiction under consideration. If an outsider joins a jurisdiction, the median voter of the enlarged jurisdiction typically differs from the former one. As a result, for some citizens, the gains from having more citizens financing the public good can be offset by the losses due to a change in the median voter. Therefore, even if Pareto improvements over the current decision exist, the majority mechanism may not pick one of them.

As we will see, whether increasing returns hold or not has important consequences for the interaction between free mobility and free entry.

\subsubsection{Superadditivity, coalition structures and efficiency}

The benefits for disjoint groups to join crucially determine group formation. A game in which each coalition is always at least as efficient as any of its partition is called superadditive. Superadditivity may be expressed as

$$
V(S) \cap V(T) \subset V(S \cup T) \text { whenever } S \text { and } T \text { are disjoint, }
$$

since $V(S) \cap V(T)$ are the payoffs that can be achieved by two coalitions $S$ and $T$ acting separately.

As for increasing returns, the decision process used within coalitions may affect superadditivity (see the analysis of a public goods economy in section 4.1). 
Under superadditivity, efficiency can always be reached by the whole group $N$. Instead, in situations in which the members of a group can all be made better off by being partitioned into a smaller self-sufficient groups, there is no reason to exclude this possibility. A coalition structure precisely takes into account the splitting possibilities.

Definition 6 A coalition structure of $N$ is a family $\left(a_{\ell}, S_{\ell}\right)_{\ell=1, \ldots, L}$ where $\left(S_{\ell}\right)_{\ell=1, \ldots, L}$ is a partition of $N$, and $a_{\ell}$ is feasible for $S_{\ell}, \ell=1, \ldots, L$.

In the economy of section 2, a coalition is a group of customers who buy the same offer, and a coalition structure amounts to a feasible configuration.

At a structure $\left(a_{\ell}, S_{\ell}\right)_{\ell=1, \ldots, L}, i$ 's utility level is equal to $u_{i}\left(a_{\ell(i)}\right)$, where as before $\ell(i)$ denotes the coalition to which $i$ belongs. So a coalition structure that is Pareto optimal among all coalition structures, called efficient, is defined by:

Definition 7 A coalition structure $\left(a_{\ell}, S_{\ell}\right)_{\ell=1, \ldots, L}$ is efficient if no other coalition structure makes everybody better off: for no coalition structure $\left(a_{\ell}^{\prime}, S_{\ell}^{\prime}\right)_{\ell=1, \ldots, L^{\prime}}$ $u\left(a_{\ell^{\prime}(i)}\right)>u\left(a_{\ell(i)}\right)$ for each $i$.

Efficiency implies efficiency within coalitions, a notion called intra-group efficiency. Formally, a structure $\left(a_{\ell}, S_{\ell}\right)_{\ell=1, \ldots, L}$ is said to be intra-group efficient if $a_{\ell}$ is Pareto-optimal for $S_{\ell}$ among the alternatives of $A\left(S_{\ell}\right)$. For example, a structure $(a, N)$ composed with a Pareto efficient decision for the whole society is intra-group efficient. Also, in a hedonic game, any structure is intra-group efficient, since a coalition has a single possible decision. As made clear by these examples, which coalitions form also matters for a structure to be efficient. Thus, efficiency is stronger than intra-group efficiency.

Superadditive cover For games that are not superadditive, allowing coalition structures enlarge the set of feasible payoffs. The superadditive cover $\hat{V}$ of the initial game $V$ precisely assigns to each group the set of payoffs that it can reach through all its coalition structures. To define $\hat{V}(T)$, note that if $T$ splits into the partition $\left(T_{\ell}\right)_{\ell=1, \ldots, L}$, it can reach any payoff in $\cap_{\ell=1, \ldots, L} V\left(T_{\ell}\right)$. Thus $\hat{V}(T)$ is obtained as the union of these payoffs over all partitions of $T$ :

$$
\hat{V}(T)=\cup_{\left\{\left(T_{\ell}\right)_{\ell=1, \ldots, L} \text { partition of } T\right\}} \cap_{\ell=1, \ldots, L} V\left(T_{\ell}\right) .
$$

Clearly, $\hat{V}$ is superadditive. Also, if $V$ is superadditive, $\hat{V}$ coincides with $V$.

On the number of groups at an efficient coalition structure Quite naturally, the number of coalitions necessary to reach efficiency depends on the underlying game. Some insight can be gained from the analysis of Guesnerie and Oddou (1987). They focus on situations in which efficiency is reached by a unique coalition, the whole society. By definition, $N$ is always as efficient as several disjoint coalitions if, for any coalition structure $\left(a_{\ell}, S_{\ell}\right)_{\ell=1, \ldots, L}$ of $N$, there is an action $b$ feasible for $N$ that makes everybody as well off, namely such that

$$
\text { for any } i \text { in } N, u_{i}(b) \geq u_{i}\left(a_{\ell(i)}\right) \text {. }
$$


This property, which is weaker than superadditivity ${ }^{26}$, is called the universal efficiency of $N$. Interestingly, Guesnerie and Oddou provide a necessary condition for universal efficiency that is easy to interpret. To introduce this condition, let us consider the simple situation where $N$ is partitioned into two sets say $S$ and $T$. Let us pick two agents, say $s$ and $t$, as "dictators" respectively in $S$ and $T$, meaning that each one chooses his preferred action respectively in $A(S)$ and $A(T)$. If $N$ is universally efficient, then surely the two dictators can find a mutually advantageous decision under the condition that the whole society forms and applies this decision. Note that this must hold whatever the partition under consideration and whatever the couple of agents that are designated as dictators. This yields the condition of binary superadditivity:

A game is binary superadditive if for any partition of $N$ into two coalitions $S$ and $T$, any $i$ in $S, j$ in $T$, $a$ in $A(S)$, and $b$ in $A(T)$ there exists $c$ in $A(N)$ such that

$$
u_{i}(c) \geq u_{i}(a), u_{j}(c) \geq u_{j}(b) .
$$

Binary superadditivity is necessary for the whole society to be universally efficient. It should be clear that the more homogeneous the population and the larger the returns to size, the more likely it is that the game will be binary superadditive.

It turns out that if the set of alternatives is one-dimensional, binary superadditivity is also sufficient for $N$ to be universally efficient. This is not true, however, if the set of alternatives is multidimensional. Then, a stronger condition called multilateral merging agreements guarantees the universal efficiency of $N$. Agreement has to be found for partitions into more than two groups. Furthermore, the maximal number of groups in these partitions increases with the dimension of the alternative space. Thus, quite naturally, the larger the dimension, the more numerous the partitions to consider, and the stronger the condition of multilateral merging agreements (we refer the interested reader to Guesnerie and Oddou (1987) for a precise definition).

Whereas this analysis treats the universal efficiency of the whole society, it can be carried out on coalitions. In particular, if the whole society is not universally efficient, finding the coalitions that are universally efficient can give an insight into the maximal number of coalitions that form at an efficient structure.

The main issue we are concerned with is whether some form of competition helps, or forces, the group $N$ to reach an efficient structure. We start by examining the pressure exercised by individuals who are free to move and join whatever existing coalition. This gives rise to the free mobility conditions. We proceed with the pressure exercised by coalitions, which gives rise to the free entry conditions. The relationships between free mobility and free entry are investigated afterwards.

\subsection{Free mobility}

Given a coalition structure, consider an individual who contemplates leaving the coalition to which he belongs. Under free mobility, this person has the

\footnotetext{
${ }^{26}$ Note that superadditivity requires each coalition to be universally efficient.
} 
opportunity to stay single or to join an existing coalition, and decides according to the expected benefits of each alternative.

The expected benefits from staying single is simply the maximum utility level over the individual's feasibility set, the individual rationality level. The expected benefits from joining a coalition are more ambiguous because they are affected by the impact that the individual expects to have on the action taken by the coalition. In most situations we are interested in, where coalitions are large and engaged in a collective activity such as the levels of public goods and their financing through taxes, an individual naturally expects to have no impact $^{27}$ : he takes the action of the coalition as "given", without attempting to change this action or considering the externality he may impose on the group members. This assumption coincides in the differentiated goods economy with consumers who are "price and product takers", as already considered. We start with this situation. For stating the definition it is convenient to introduce a fictitious action $a_{0}$, where $u_{i}\left(a_{0}\right)$ is set equal to $i$ 's individual rationality level.

Definition 8 A coalition structure $\left(a_{\ell}, S_{\ell}\right)_{\ell=1, \ldots, L}$ satisfies free mobility if

$$
\text { for each } i, u_{i}\left(a_{\ell(i)}\right) \geq u_{i}\left(a_{k}\right) \text { any } k=0,1, \ldots, L \text {. }
$$

Structures that satisfy free mobility typically exist, and in fact are numerous. Furthermore most of them are inefficient, and even intra-group inefficient. To see this, consider a structure $(a, N)$ where only the whole group $N$ forms. Free mobility is satisfied if action $a$ gives to each individual at least his rational utility level : for each $i, u_{i}(a) \geq u_{i}\left(a_{0}\right)$. In most problems of interest, there are many such actions. Moreover, most of them are Pareto dominated by another feasible action for $N$, in which case the structure is not only inefficient but also intra-group inefficient.

We now consider the case where an individual has an impact on the decision taken by the coalition he contemplates joining. If the individual is aware of this impact, free mobility should be defined in the line of a Nash equilibrium. We restrict here to hedonic games. In these games the payoff accruing to a player who joins a coalition is uniquely defined, hence can be anticipated without ambiguity ${ }^{28}$. A coalition structure, which simply amounts to a partition $\left(S_{\ell}\right)_{\ell=1, \ldots, L}$ of the society $N$, is Nash stable if setting $S_{0}=\emptyset$

$$
\text { for each } i, u_{i}\left(S_{\ell(i)}\right) \geq u_{i}\left(S_{k} \cup i\right) \text { for any } k=0,1, \ldots L \text {. }
$$

For the same reasons as for free mobility, Nash stability does not imply efficiency, or even intra-group efficiency.

The intuition for why single individual moves are far from ensuring efficiency is quite clear: exploiting efficiently returns to collective action requires some

\footnotetext{
${ }^{27}$ To make sense an action must be "anonymous", which means that the action taken by a coalition can be applied without ambiguity to an outsider. This is the case in all the examples we have seen so far.

${ }^{28}$ In games that are not hedonic, because no decision mechanism is fixed within a coalition for instance, the reaction to the arrival of a new member may not be uniquely defined. Hence individuals' incentives to move are affected by their conjectures about these reactions. This difficulty does not arise in a hedonic game.
} 
coordination. This inefficiency result however may not be so problematic, for, because of these returns, coordinated moves, and not only moves by single individuals, are likely to be at work. We examine now these coordinated moves and whether they restore efficiency.

\subsection{Free entry coalition structures}

Coordinated actions within a group are presumably the driving forces that explain competition across groups. These forces can be modeled through the standard blocking conditions properly extended to coalition structures.

Definition 9 A coalition structure $\left(a_{\ell}, S_{\ell}\right)_{\ell=1, \ldots, L}$

- is blocked by coalition $S$ if there is an action b feasible for $S$ that makes every member of $S$ better off :

$$
\text { for some } b \text { in } A(S) u_{i}(b)>u_{i}\left(a_{\ell(i)}\right) \text { each } i \text { in } S \text {, }
$$

- satisfies free entry (or is stable under free entry) if it is blocked by no coalition, or equivalently if the payoff vector $\left(u_{i}\left(a_{\ell(i)}\right)\right)_{i \in N}$ belongs to the core of the superadditive cover $\hat{V}$ defined by equation (5).

Under free entry, any group can form, and does form, if it is in the members interests. It is important to note that, even in the absence of spillovers, the full organization of the society is relevant to assess its stability: the opportunity of a move for the members of a given coalition is determined by the organization of outside agents. Stability under free entry corresponds to the standard stability notion of the core applied to the situation in which the society can partition itself. We draw three consequences from this simple remark.

Efficiency Although straightforward, the first consequence is nevertheless important. Because a core outcome is always Pareto efficient, a coalition structure that satisfies free entry is efficient among all feasible coalition structures. As a result, several coalitions form only if the splitting of the society into smaller groups is triggered by efficiency forces, that is in non superadditive games ${ }^{29}$.

In settings in which a divisible good can be transferred across coalitions, balanced transfers do not lead to a Pareto improvement either, as argued in Theorem 2: A coalition structure that satisfies free entry is Pareto efficient among coalition structures with cross subsidies.

Efficiency results from free entry, but not from alternative coalition threats. To see this, consider the model of formation of nations, as described in example 3 of section 3.1. The location of a government within a nation is chosen according to majority rule, which gives rise to a hedonic game. In this game, a new "nation" forms and blocks if, given the majority winner within this new nation, each member is made better off than under the current structure. A stable structure can be shown to exist. It is efficient in the hedonic game, that is, among all structures in which majority rule within a country is used.

\footnotetext{
${ }^{29}$ Exploiting this result, Demange and Guesnerie (1997) provide a set up in which the whole society may collaborate without being blocked.
} 
Alesina and Spolaore (1997) consider a different notion of stability, or equivalently of secession. They assume that a new "nation" forms if the modification is approved by a majority in each of the existing countries affected by the change of the borders. It is important to understand that majority rule here is used at two distinct levels: at a first level, within a country for choosing the location of the government, and at a second level for modifying, and destabilizing, the borders of the countries. Therefore, in contrast to blocking, unanimity within a destabilizing coalition is not required, which makes secession easier. As a result, inefficiency results: too many nations form ${ }^{30}$.

It should be noted that the rules that usually apply to form a new jurisdiction (the second level) differ from the rules that apply to take decisions within each jurisdiction (the first level); indeed they are much more stringent than simple majority. Even though unanimity may not be required to form a new jurisdiction, the blocking condition is likely to be closer to normal practice.

Existence The second consequence that can be drawn from the core analysis is rather negative. As has been well known since Shapley (1967), the core of a superadditive game may be empty. Thus, without further restrictions, free entry coalition structures may not exist. The previous section has shown that under some restrictions on preferences, the intermediate preferences property, existence is obtained. Free entry may also be restricted in order to restore existence. How serious are these restrictions ? Section 3.6 examines this question.

Fee entry without increasing returns. Finally, the third consequence looks a priori more technical but is very important in many applications. Increasing returns to coalitions are not at all necessary for a free entry coalition structure to exist. To see this, note that a free entry coalition structure exists when the core of the superadditive cover $\hat{V}$ is nonempty, which is independent of whether the sets of payoffs $V(S)$ increase with $S$. Why do we stress this point? It turns out that, without increasing returns, a coalition structure that is stable under free entry may not satisfy free mobility. If some individuals do not belong to the group they prefer, is a free entry coalition structure stable in some reasonable sense? We investigate this question now.

\subsection{Free mobility, free entry, and increasing returns}

It is first important to understand that free entry does not a priori imply free mobility. The reason is that, under free mobility, an individual contemplates joining a standing coalition without evaluating the welfare of the insiders. Therefore, an individual $i$ may want to join a coalition $S_{\ell}$ even if the members of $S_{\ell}$ do not benefit from the newcomer. This means that, even if the enlarged coalition formed with the insiders and the newcomer, $S_{\ell} \cup i$, does not block, $i$ may want to join $S_{\ell}$ : free entry does not imply free mobility.

If feasible sets are increasing however, free mobility most often follows from free entry. The argument is as follows. Consider an individual who is strictly

\footnotetext{
${ }^{30}$ Again, inefficiencies are here assessed in the game that takes the majority rule within a country (the first level) as a constraint.
} 
better off by joining an existing group under the current action taken by that group. By keeping the action unchanged, the insiders are equally well off. Therefore, the enlarged coalition formed with the insiders and the newcomer blocks in a "weak" sense. Under a smoothness assumption, the action can be slightly modified so that every member is strictly better off, thus contradicting free entry. This gives the following property.

Assume that feasible sets are increasing. Under smooth payoffs, a coalition structure that satisfies free entry satisfies free mobility as well.

What can be said about the stability of a free entry coalition structure if feasible sets are not increasing ? In a situation where a group can prevent newcomers to join it, it will use this possibility whenever it is in the members' interest. Consider for example that an individual can join a group only if each member of that group agrees. Free mobility then amounts to free mobility under agreement. We describe this in the simple set up of a hedonic game ${ }^{31}$

A coalition structure $\left(S_{\ell}\right)_{\ell=1, \ldots, L}$ satisfies free mobility under agreement if for each $i$, each $S$ one of the existing community $S_{\ell}, \ell=1, \ldots, L$ or $S=\emptyset$ it is not true that

$$
u_{i}(S \cup i)>u_{i}\left(S_{\ell(i)}\right) \text {, and } u_{j}(S \cup i) \geq u_{j}(S) \text { for any } j \text { in } S .
$$

It should be clear thatfree mobility is drastically restricted. Free mobility under agreement simply requires that no group $S_{\ell} \cup i$ weakly blocks. Therefore, arguing as above, one straightforwardly obtains:

Under smooth payoffs, a coalition structure that satisfies free entry satisfies free mobility under agreement as well.

Therefore, even if feasible sets are not increasing, a free entry structure can indeed be considered stable provided that coalitions are able to screen potential newcomers and possibly to forbid them to join ${ }^{32}$. In a variety of situations however, a group is unable to exclude some individuals, at least without bearing a cost. For example, exclusion policies based on identity only or on some criteria may be forbidden. Also a group, especially a large one, is often poorly informed on the newcomers' characteristics. Section 4.2 investigates this situation.

\subsection{Restrictions on free entry or on preferences}

We have seen that free entry structures do not necessarily exist. We examine here which restrictions on free entry or on preferences help to restore existence.

\footnotetext{
${ }^{31}$ For various definitions and discussions of free mobility in hedonic games see Bogomolnaia and Jackson (2002)). Also immigration rule under which a jurisdiction accept immigrants can be seen as a form of restriction to free mobility. See the analysis of various immigration rules by Jehiel of Scotchmer (2001).

${ }^{32}$ The possibility of forbidding entry can be reasonably assumed in a small population, as for example in the analysis of partnerships of Farell and Scotchmer (1988). See also chapter 13 in this volume, which analyzes the formation of coalitions of countries (a "player" is a country). Once a coalition of countries is formed, each country within the coalition decides on its own contribution to a growth enhancing public good. Due to difference in endowments across countries, increasing returns to coalitions fail. Assuming the possibility for a coalition of countries to exclude a (poorer) country makes sense.
} 
Restrictions on free entry If there are limits to free entry, stable coalition structures should be more likely to exist. The question is to which extent must free entry be limited to guarantee stability? We investigate here ${ }^{33}$ the situation where some coalitions simply cannot form. Such restrictions naturally arise in some settings. Let us give two examples.

- Marriage and assignment games. The society is partitioned into two groups, say men and women, buyers and sellers, colleges and students. Let us consider for example the marriage problem (Gale and Shapley (1962)). Only singletons and pairs consisting of one man and one woman can form. A marriage defines a set of couples, each individual having at most one spouse. The marriage is stable if (1) no new couple can form in which both the man and the woman prefer each other to their standing partner (no couple blocks) (2) no person would prefer to stay single (no individual blocks). Gale and Shapley show that a stable marriage always exists.

- Connected coalitions in a network. If communication among individuals is constrained by a network, quite naturally only the coalitions that are connected in the graph can form ${ }^{34}$. If the network is a simple path, individuals are completely ordered, and only intervals can form: a consecutive game is obtained as considered by Greenberg and Weber (1993). More generally, if the network is a tree, individuals are partially ordered, as in Demange (1994).

How much must free entry be restricted to guarantee stability regardless of the preferences? This leads to the following definition, as introduced by Kaneko and Wooders (1982).

Definition 10 Given a set $\mathcal{C}$ of coalitions of $N$, a coalition structure is said to be $\mathcal{C}$-stable if it is blocked by no coalition in $\mathcal{C}$. The set $\mathcal{C}$ guarantees stability if any game admits a $\mathcal{C}$-stable coalition structure.

Clearly, the more coalitions that are allowed to block (i.e. the larger the set $\mathcal{C}$, ) the stronger the stability concept. If all coalitions can form, we know that stable coalition structures may not exist. At the opposite, if only the whole society and the singletons are allowed to block, $\mathcal{C}$-stability coincides with Pareto optimality and individual rationality : stability is guaranteed.

Consider the preceding examples. Stability is guaranteed in marriage games. In networks, stability is guaranteed if the network is a line or more generally is a tree, but fails if the network contains a cycle. There are no other known examples that are associated to meaningful contexts ${ }^{35}$. One can say however that free entry must be drastically restricted to guarantee stability. To understand

\footnotetext{
${ }^{33}$ Other types of restriction to entry could be considered. For example a new group can form only if it is a subset of an existing group (internal stability within a group) or if it is the union of two existing groups. We are not aware of any general result under such restrictions.

${ }^{34}$ Kalai, Postlewaite and Roberts (1978) were the first to analyze stability under this restriction. In an exchange economy they perform some comparative statics on the set of stable allocations by allowing the graph to vary. Because in an exchange economy the core is always nonempty, their concern clearly differs from ours.

${ }^{35}$ There are mathematical characterizations. Thanks to standard results on balanced games one easily shows that $\mathcal{C}$ guarantees stability if and only if any balanced family of sets in $\mathcal{C}$ contains a partition. Kaneko and Wooders derive a characterization in terms of the extreme solutions to a system of linear equations.
} 
this, the following condition, based on triples of coalitions, is helpful. Let us define a Condorcet triple as three coalitions that intersect each other and whose overall intersection is empty:

$$
S_{i}, i=1,2,3 \text {, with } S_{i} \cap S_{j} \neq \emptyset \text { and } S_{1} \cap S_{2} \cap S_{3}=\emptyset .
$$

One can show that if a set $\mathcal{C}$ of coalitions guarantees stability, then it contains no Condorcet triple. The argument is by contradiction. Take a family $\mathcal{C}$ that contains a Condorcet triple. Consider the transferable utility game with characteristic function $V: V(N)=1, V(S)=b$ if $S$ is a strict subset of $N$ that contains at least one $S_{i}, i=0,1,2$, and $V(S)=0$ otherwise. For $b$ smaller than 1 the game is superadditive but, for $b$ strictly smaller than $2 / 3$, any feasible payoff is blocked by one of the $S_{i}$. Note that stability fails in numerous games. Hedonic games could be defined as well.

The absence of Condorcet triples is a strong restriction (which is furthermore not sufficient). Therefore, the lesson that can be drawn from this analysis is that, without restrictions on preferences, the blocking power of coalitions, or equivalently their possibility to form, must be quite drastically restricted in order to guarantee stability. We now examine restrictions on preferences.

Restrictions on preferences Under some restrictions on preferences, only the blocking possibilities of a restricted set of coalitions matter. Therefore, the previous approach is very helpful to get some insight on the kind of existence results that can be obtained by restricting preferences.

For example, why does a sustainable configuration exist under a one-dimensional intermediate preferences family (Theorem 1) ? Thanks to the one-dimensional assumption, individuals can be ordered according to their characteristics. Therefore, there is a coalition structure that is formed with "intervals" of individuals and that no interval blocks. Increasing returns to coalitions and intermediate preferences then imply that this coalition structure is blocked by no coalition at all. The same argument can be used if the characteristic belongs to a tree. This yields the following result, which extends Theorem 1 (Demange 1994).

Let preference characteristics belong to a tree. Under intermediate. preferences and increasing returns to coalitions, a coalition structure that is stable under free entry exists.

Now consider characteristics to be multi-dimensional, bi-dimensional for instance, and still assume intermediate preferences. By a similar argument as the one just presented, a coalition structure formed with convex sets that is not blocked by a convex set satisfies free entry. However, such a structure may not exist because the family of convex sets does not guarantee stability: in a two dimensional space, it is easy to find a Condorcet triple formed with convex sets. Similarly, given a graph with a cycle, the family of connected sets does not guarantee stability. This explains why a stable structure does not exist for a large set of parameters in the example of destructive competition in section 2.3.1. This leads us to our second point.

That there are very few sets $\mathcal{C}$ that guarantee stability suggests that free entry coalition structures do not exist except under specific assumptions. To 
be more precise, let us take the feasible sets as the primitives, and assume preferences to be unknown. If the class of preferences is not rich, a stable coalition structure may exist without any restriction on the restrictions on the set of admissible coalitions ${ }^{36}$. If the class of possible preferences is rich enough, however, surely there are some profiles for which no stable coalition structure exists. This may provide a rationale for organizations such as tree-hierarchy structures that restrict the set of blocking coalitions (Demange (2004)).

\section{Public decision rules and mobility}

Some of the central questions regarding public decisions can be discussed through the tools introduced in the previous section. Choosing public goods levels and their financing, or deciding on a redistributive policy are subject to objections stemming from part of the population. Also, these decisions are affected by the ability of individuals to move to another jurisdiction or to another country.

To discuss these issues we first introduce a very simple economy in which a public good is financed through a tax system. Basic properties such as superadditivity and increasing returns to coalition are shown to depend crucially on the fiscal tools available to a government, and on the decision process used. That fiscal tools affect the efficiency frontier is well known from tax theory. It is less well known that fiscal tools affect stability as well. In particular, if redistributive policies are implemented, increasing returns to coalition may fail : an individual may have a negative impact on a group he joins. Then free mobility does not follow from free entry and must be explicitly taken into account. Alternative equilibrium approaches to address the interaction between free mobility and free entry are discussed.

\subsection{Fiscal tools, decision rules and increasing returns}

We consider an economy ${ }^{37}$ with two private goods, a consumption good and labor, and a public good. Both consumption and public goods are produced from labor through constant returns to scale technologies. Up to a normalization, a unit of labor produces respectively $c$ units of consumption and 1 unit of public good. Preferences over private and public goods are separable, represented for a $\theta$-agent by :

$$
u(x, l, y, \theta)=v(x, l, \theta)+w(y)
$$

where $x, l, y$ are respectively the amounts of consumption good, labor and public good, and standard assumptions are made on $v$.

From the efficiency point of view, the whole society should join, leaving access to the public good to each agent. If personalized prices are implementable without cost, the incremental value generated by an individual joining a coalition increases as the coalition grows. As a result, due to a "snowballing" or

\footnotetext{
${ }^{36}$ For an example in a hedonic set up, see the top cycle property defined by Banerjee, Konishi and Sonmez (2001).

${ }^{37}$ The analysis here is close to Guesnerie and Oddou (1981).
} 
"band-wagon" effect, there are many stable outcomes ${ }^{38}$, among them the Lindahl equilibrium (see Champsaur (1975) and Demange (1987) respectively with and without transferable utility). Most often however, public goods are financed through taxes, because personalized prices cannot be charged, due e.g. to a lack of information. Tax systems restrict the possibilities of distributing the potential gains derived from public goods within the population.

Tax system and feasible sets We consider here tax systems that are composed of a nonnegative tax on the consumption good, denoted by $t$, and a uniform lump sum tax, possibly negative, denoted by $r$ in terms of units of labor. The overall tax burden for an individual is the sum of the amount of taxes on consumption and the lump sum $r$. Accordingly, a system $(t, r)$ with $r$ is negative is described as redistributive because some individuals may be net receivers. To handle constraints such as the absence of redistribution, or $r$ not too large, we assume that $r$ must be chosen in $[\underline{r}, \bar{r}]$. Thus, according to the previous remark, redistribution is excluded if $\underline{r} \geq 0$.

The feasibility sets associated to these tax systems are determined as follows. Let a group of citizens $S$ decide to form a community. If the tax system $(t, r)$ is chosen by $S$ a unique equilibrium is derived as follows. Since returns to scale are constant, the competitive production price for the private good is equal to its constant average cost $c$, and the consumption price is $c+t$. Thanks to separable preferences, the consumer's demand, $x(t, r, \theta), l(t, r, \theta)$, maximizes :

$$
v(x, l, \theta) \text { over }(x, l) \text { such that }(c+t) x \leq l-r .
$$

Thus, the budget surplus for community $S$ can be expressed as

$$
\pi(y, t, r, S)=\sum_{i \in S} t x\left(t, r, \theta_{i}\right)+r|S|-y .
$$

Budget balance, $\pi(y, t, r, S)=0$ determines the level of public good. Furthermore, the market for consumption good clears through an appropriate production scale, and by Walras law the labor market also clears. Thus, given $(y, t, r)$ that satisfies budget balance for community $S$, a payoff vector to each member of $S$ is derived. Therefore, if no decision process is further specified, a group of citizens $S$ can choose any policy that is budget balanced :

$$
A(S)=\{(y, t, r) \text { that satisfies } t \geq 0, r \in[\underline{r}, \bar{r}], \pi(y, t, r, S) \geq 0\} .
$$

The above feasible set is quite large. If further constraints on the tax system and/or the decision process within a community are added, the feasible sets are subsets of $A($.$) . Therefore, each set of constraints gives rise to a different$ game. We investigate the properties of the induced game, superadditivity and increasing returns, starting with the case where no decision process within a community is fixed and proceeding with the majority rule.

- No fixed decision rule.

Without fixed rule, feasible sets $A($.$) are given by (8).$

\footnotetext{
${ }^{38}$ In technical terms, the game is not only superadditive but also convex, as defined first by Shapley (1971).
} 
Increasing returns. Monotonicity properties depend on whether redistribution is possible. Consider whether feasible sets are increasing, that is, whether a policy $(y, t, r)$ feasible for $S$ remains feasible if newcomers join it. Clearly, if the budget $\pi$ given by equation (7) increases with $S$, feasible sets are increasing. The amount of taxes collected from consumption can only increase with $S$ ( $t$ is nonnegative). Therefore, without redistribution $(\underline{r} \geq 0) \pi$ is non decreasing with $S$ so that feasible sets are increasing. Instead, with a redistributive policy $(r<0)$ the budget may decrease as the population is enlarged. Then feasible sets and returns to coalitions may not be increasing. The argument should be clear: the arrival of individuals who receive a positive net payment through the tax system (individuals with low consumption in the private good) has a negative impact on the budget and hurts the insiders. A similar argument applies if a proportional income tax is raised : newcomers whose income is low enough to be net tax receiver impose a negative externality on incumbents.

Superadditivity. In a situation in which personalized prices cannot be charged, efficiency gains are not freely distributed among individuals. As a consequence, the game is not necessarily superadditive, and the formation of several groups cannot be excluded even from a Pareto point of view. This is illustrated by the following example, which has nothing pathological.

Example Preferences are given by $\theta v(x, l)+w(y)$. Consider only a lump sum $\operatorname{tax} r$ so that an individual faces the budget constraint $c x \leq l-r$. Defining $U(r)=\max _{x} v(x, c x-r)$ the indirect utility over the consumption good, a $\theta$-individual derives the utility level $\theta U(r)+w(y)$ from public good level $y$ and $\operatorname{tax} r$.

Individuals' characteristics take two values only $\theta_{\ell}, \ell=1,2$. Denote by $N_{\ell}$ the group of individuals with characteristic $\theta_{\ell}$, and by $n_{\ell}$ its cardinality. Assume that $N_{\ell}$ forms. Since $N_{\ell}$ contains identical individuals, it optimally chooses the level of tax, $r_{\ell}$, that maximizes $\theta_{\ell} U(r)+w\left(n_{\ell} r\right)$. Now if the whole society $N$ forms and chooses $r$, individuals in $N_{\ell}$ are better off if the inequality

$$
\theta_{\ell} U(r)+w(n r) \geq \theta_{\ell} U\left(r_{\ell}\right)+w\left(n_{\ell} r_{\ell}\right)
$$

holds. The set of $r$ that satisfies the above inequality is an interval $I_{\ell}$ that contains $r_{\ell}$. All individuals can be made better off by forming $N$ if the intersection $I_{1} \cap I_{2}$ is non-empty (then $N$ is universally efficient). The intersection however may be empty, leading to a non superadditive game. With equal groups for example, the game is not superadditive if the difference between the valuations $\theta_{\ell}$ for the private good is large enough.

- Majority rule. We examine now the case where the tax system within each community is chosen under majority rule. To ensure the existence of a majority winner, assume that the tax parameter on which individuals vote is one-dimensional. For instance, they vote on the poll level $r$ only. Returns to coalitions may fail to be increasing. To see this, consider the previous example. Inside the homogeneous group $N_{\ell}$, the majority winner is $r_{\ell}$. Now, assume $N_{1}$ to be more numerous than $N_{2}$. Under simple majority, society $N$ chooses the decision $r_{1}^{\prime}$ that maximizes $\theta_{1} U(r)+w(n r)$. Even if there are decisions that could make everybody better off, the majority may not choose one of them: 
even if $I_{1}$ and $I_{2}$ intersect, $r_{1}^{\prime}$ does not necessarily belong to the intersection.

To conclude, this section has shown that the redistribution performed by jurisdictions most often generates negative externalities among citizens. Increasing returns to coalitions fail because of the anonymity of the tax policy, anonymity that may be due to various factors, such as poor information. If a coalition could screen the newcomers who exert a negative effect on insiders, the difficulty would not be so serious as we have seen in section 3.5. In most countries, citizens are allowed to move freely from one community to another, and information is poor. In such a context the interaction between free mobility and free entry is not trivial. Indeed, similar difficulties arise in defining competition in insurance markets where negative externality stems from adverse selection (Rothschild and Stiglitz (1976)). We present first a possible definition of blocking in a general framework and then discuss it in our simple public good economy.

\subsection{Blocking under negative externality}

Under negative externalities and free mobility, a group setting its policy has to take into account not only the welfare within the group but also the attractiveness the policy may have on outsiders. Also, a tentative blocking coalition must derive some information on the characteristics of its members in order to assess the feasibility of a secession. A natural point of view is to base this information on self selection arguments. In this approach, the possible moves of the citizens are derived in assessing their incentives to do so given the utility levels at the candidate equilibrium. This leads to the following definition of credible blocking.

Definition 11 Coalition $T$ credibly blocks via $b$ the structure $\left(a_{\ell}, S_{\ell}\right)_{\ell=1, \ldots, L}$ if the action $b$ is feasible for $T$ and if $T$ is the whole set of individuals who strictly prefer $b$ to the current policy of the community to which they belong:

$$
\begin{aligned}
& u_{i}(b)>u_{i}\left(a_{\ell(i)}\right) \text { for any } i \text { in } T \\
& u_{i}(b) \leq u_{i}\left(a_{\ell(i)}\right) \text { for any } i \text { not in } T .
\end{aligned}
$$

Credible blocking entails two conditions: first, as usual, each member of the blocking coalition has incentives to join, second, no outsider wants to do so.

What kind of process enables a coalition to block ? This question is important because we are interested in situations in which individuals cannot be screened. Consider the public good model. Let policy $(y, t, r)$ be proposed. Assume that citizens (or at least a city manager) rationally expect the coalition $T$ of individuals who are attracted by this proposal. Then coalition $T$ credibly blocks if the policy is budget feasible for $T$, i.e. if $\pi(y, t, T) \geq 0$, where $\pi$ is defined by (7). Otherwise, if $\pi(y, t, T)<0$, a tâtonnement process can be contemplated, for example as follows: the level of public goods is adjusted down to $y^{\prime}$ so as to satisfy the budget constraint $\pi\left(y^{\prime}, t, T\right)=0$, which leads to a new set $T^{\prime}$ and so on. If the process converges to a nonempty coalition, a credible coalition is obtained. Note that only the information on the distribution of characteristics is necessary, not the identity of individuals. 
Similar ideas have been used in various contexts with asymmetric information : in a credit market with two types of individuals (Boyd and Prescott (1986)), in an income taxation model à la Mirlees with non linear income tax schedules (Berliant (1992)), in a mechanism design set-up (Demange and Guesnerie (2001)), and finally within a local public good economy subject to congestion with homogeneous consumers (Conley and Konishi (2002)). To our knowledge, neither the existence nor the properties of stable outcomes have been analyzed in a general framework. Actually, the analysis of blocking under asymmetric information is still in progress ${ }^{39}$. Finally, self selection arguments are also implicit in the model with redistribution analyzed by Epple and Romer (1991) that we discuss now.

\subsection{Free mobility with a fixed number of jurisdictions}

As seen in section 4.1, redistribution within jurisdictions typically generates negative externalities. Epple and Romer precisely address the impact that free mobility may have on communities' decisions. To simplify we present here their approach in the public good economy of the previous section. Distinctive features from the analysis of competition across groups carried out in section 3 are that

(1) the number of communities is fixed, say a community is associated with a given geographical zone with fixed boundaries

(2) stability is assessed from the point of view of insiders : a new policy may destabilize a community only if the current inhabitants agree to it, possibly taking into account the population changes triggered by the new policy.

To simplify, let us restrict to two communities. A structure is given by the set of inhabitants in each community together with a budget balanced policy : $\left(a_{1}, S_{1}\right),\left(a_{2}, S_{2}\right)$, where $a_{\ell}=\left(y_{\ell}, t_{\ell}, r_{\ell}\right)$ satisfies $\pi\left(y_{\ell}, t_{\ell}, r_{\ell}, S_{\ell}\right)=0, \ell=1,2$. To make the model more realistic, we allow individuals to be concerned with some intrinsic exogenous characteristics of a community : $u_{i}$ is function of both the decision $a$ taken by a community and the community "identity" $l$. Free mobility says as usual that no citizen wants to move to the other community, taking both policies as given :

$$
\text { for any } i, u_{i}\left(a_{\ell(i)}, \ell(i)\right) \geq u_{i}\left(a_{k}, k\right), k=1,2 \text {. }
$$

Citizens in a community vote on the tax parameters $(t, r)$, the level of the public good being determined by budget balance over the population within the community. Decisions are taken under $q$-majority, where $q$ is between one half (majority) and one (unanimity).

\footnotetext{
${ }^{39}$ Wilson (1978) first tackles the problem by defining several concepts according to the communication system used by a coalition if it forms. The incentives to abide by the communication system are however not considered : individuals ex ante commit to a given communication system. Since this pioneering work, another line of research studies the ex ante coalitional stability of such mechanisms, which are implemented at the interim stage (see the special issue of Economic Theory 2001).
} 
To form a (voting) equilibrium, a structure must satisfy two conditions: first free mobility, and second in each community no alternative policy destabilizes the current policy according to the voting rule. The second condition means that no policy gets a proportion larger than $q$ of the votes within the community against the current policy. The difficulty is to predict how citizens vote. Indeed, different equilibrium outcomes may arise according to the level of voters' farsightedness. When a voter in a given community compares different policies, he may or may not take into account the impact that each policy has on the composition of the population living in the community. Let us start with "myopic" voters.

A voter is myopic if he assumes that the composition of the population is unaffected by policy decisions and derives the feasible policies accordingly: living in a community with population $S$ and contemplating another tax decision $(t, r)$ he expects the level $y$ of public good to be given by budget balance applied to population $S$. This gives the following definition.

Definition 12 A two communities structure $\left(a_{1}, S_{1}\right),\left(a_{2}, S_{2}\right)$ forms an equilibrium with myopic voters if it satisfies free mobility and

(no destabilizing policy) for each community $\ell=1,2$ : there is no $b=(y, t, r)$ with $\pi\left(y, t, r, S_{\ell}\right) \geq 0$ that is preferred to $a_{\ell}$ by a proportion of the population in $S_{\ell}$ larger than $q$.

Under unanimity, a destabilizing policy is simply a policy that Pareto dominates the current policy of a community. Without unanimity, destabilizing is easier. Therefore, at an equilibrium, surely each jurisdiction chooses a Pareto efficient decision for itself. Accordingly, both intra-group efficiency and free mobility must be met at equilibrium (and are even sufficient under unanimity).

In assessing stability, the various policies that are considered as destabilizing do not account for free mobility : if an alternative policy $b$ feasible for $S_{\ell}$ were indeed implemented, some outsiders might immigrate to community $\ell$, and some citizens might leave it (the latter case can happen if $q<1$ because then some citizens in $\ell$ may be worse off under $b$ than under $a_{\ell}$ and so might prefer to move to the other community). These moves altogether affect the feasibility of $b$, which has to be adjusted. Voters, even slightly farsighted or "sophisticated", should be aware of the interaction between a policy and the incentives of the citizens to move.

A voter is sophisticated if he takes into account population moves : he forms some expectations on how the citizens of both communities react to a policy change. Actually it is not that easy to define such expectations. In what follows, individuals base their expectations on self-selection arguments, as introduced in the previous section. Because the intrinsic characteristics of a community matter, credible blocking has to be adjusted as follows.

Given a structure $\left(a_{1}, S_{1}\right),\left(a_{2}, S_{2}\right)$, coalition $T$ credibly blocks via $b=(y, t, r)$ community $\ell$ if $\pi(y, t, r, T) \geq 0$ (feasibility), and $T$ contains all individuals who are better off to live in community $\ell$ under policy $b$ than under their current situation:

$$
\begin{aligned}
& u_{i}(b, \ell)>u_{i}\left(a_{\ell(i)}, \ell(i)\right) \text { for any } i \text { in } T \text { and } \\
& u_{i}(b, \ell) \leq u_{i}\left(a_{\ell(i)}, \ell(i)\right) \text { for any } i \text { not in } T .
\end{aligned}
$$


Since individuals care about the intrinsic characteristics of a community, a coalition $T$ may credibly block one community but not the other one (the utility levels on the left hand side depend on $\ell$ ). Without intrinsic characteristics we fall back on definition 11 of credible blocking.

Definition 13 A two communities structure $\left(a_{1}, S_{1}\right),\left(a_{2}, S_{2}\right)$ forms an equilibrium with sophisticated voters if it satisfies free mobility and

(no destabilizing credible blocking) for each community $\ell=1,2$ : there is no $(b, T)$ that credibly blocks community $\ell$ and such that $b$ is preferred to $a_{\ell}$ by $a$ proportion larger than $q$ of the population of $S_{\ell}$.

A free mobility structure that is not credibly blocked at all is clearly an equilibrium. But credible blocking is allowed at equilibrium. If $(b, T)$ credibly blocks $\ell$, all citizens of community $\ell$ who prefer $b$ to the current policy $a_{\ell}$ belong to $T$ (by definition of credible blocking). Thus, $(b, T)$ is destabilizing only if $S_{\ell} \cap T$ contains more than the proportion $q$ of the population of $\ell$. Under unanimity, this means that each current inhabitant of $\ell$ is better off.

The no destabilizing condition can be interpreted as follows. Let $(t, r)$ be an alternative proposed to community $\ell$. Current citizens in $\ell$ forecast a level of public expenditures $y$ together with a set $T$ formed by the individuals who would live in $\ell$ if $(y, t, r)$ were feasible and implemented. The alternative is destabilizing if it defeats the current policy in $\ell$ under $q$-majority when the current citizens $S_{\ell}$ vote according to these expectations. An equilibrium is obtained if there is no alternative for which this occurs.

How does each equilibrium concept relate to each other and with stability under free entry ? Comparisons are clearly much easier when the unanimity rule is used, so let us restrict to $q=1$. The relationships are clear under increasing returns to coalitions. First, an equilibrium with sophisticated voters is surely an equilibrium with myopic voters: if a new policy destabilizes the current policy with myopic voters, it makes every inhabitant better off when applied to the current population; if the new policy attracts citizens of the other community, under increasing returns to coalitions, sophisticated voters would a fortiori destabilize the current policy (note that the argument is not valid under simple $q$ majority, because sophisticated voters take into account that some inhabitants may leave the community). Second a structure that satisfies free entry is surely an equilibrium with sophisticated voters: under free entry, an entire new group may be formed without the agreement of the inhabitants who are not members of this new group. These simple results suggest that stability is the good concept when there are increasing returns to coalitions. However, this assumption is clearly too strong in the problems we are considering.

In a model with redistribution, the adverse selection phenomena come up naturally. They are also present in more subtle ways through price effects in other models as well. In a quite similar model, Epple, Romer, and Sieg (2001) consider a fixed number of communities differing a priori only in the (exogenous) housing service offer function. A posteriori communities also differ in the chosen level of tax on housing service, land, the level of public good, and the equilibrium housing price. Interestingly, Epple et al provide an empirical analysis using data from the Boston Metropolitan area and conclude that voters are indeed quite 
sophisticated. It would be interesting to perform similar analyses on different policy issues or in different countries.

\section{Conclusion}

This chapter has shown that the standard cooperative game theory is useful for analyzing the formation of groups. In particular it has displayed conditions under which competitive pressures exercised by individuals and groups lead to an efficient and stable organization. On the theoretical side, a major failure of the approach is its current inability to handle situations under negative externalities. Another open problem is the definition of an equilibrium concept that covers situations in which heterogeneity among individuals is richer, as arises if individuals differ along several dimensions. Finally, it would be of interest to test the theory in the situations in which the conditions under which stability can be reached are likely to be met.

\section{References}

Alesina, Alberto and Enrico Spolaore (1997), "On the Number and Size of Nations," Quarterly Journal of Economics 112, 1027-1056.

Aumann, Robert and Jacques Dreze (1974), "Cooperative Games with Coalition Structures," International Journal of game theory 3, 217-237.

Banerjee, Santanu, Hideo Konishi and Tayfun Sonmez (2001), "Core in a Simple Coalition Formation Game," Social Choice and Welfare 18, 135-153.

Baumol, William J., John C. Panzer and Robert D. Willig (1982), Stable markets and the theory of industry structure, New York: Harcourt Brace Jovanovich.

Berliant, Marcus (1992), "On income taxation and the core," Journal of Economic Theory 56, 121-141.

Bewley, Truman F., (1981), "A Critique of Tiebout's Theory of Local Public Expenditures," Econometrica 49, 713-740.

Bloch, Francis (2004), Group and network formation in industrial organization, Chapter 9 this volume.

Bogomolnaia, Anna and Matthew O. Jackson (2002), "The Stability of Hedonic Coalition Structures," Games and economic behaviour 38, 201-230.

Boyd, John H. and Edward C. Prescott (1986), "Financial intermediary coalitions," Journal of Economic Theory 38, 221-232.

Buchanan, James M. (1965), "An economic theory of clubs," Economica 33, $1-14$.

Champsaur, Paul (1975), "How to Share the Cost of a Public Good ?" International Journal of Game Theory 4, 113-129.

Conley, John P. and Hideo Konishi (2002), "Migration-proof Tiebout equilibrium: existence and asymptotic efficiency," Journal of Public Economics 86, 
243-262.

Conley, John P. and Stephen Smith (2003), Coalitions and clubs: Tiebout equilibrium in large economies Chapter 8 , this volume.

Currarini, Sergio (2003), "On the Stability of Hierarchies in Games with Externalities," mimeo, Università di Venezia.

Demange, Gabrielle (1987), "Nonmanipulable Cores," Econometrica 55, 10571074.

Demange, Gabrielle and Dominique Henriet (1991), "Sustainable Oligopolies," Journal of Economic Theory 54, 417-428.

Demange, Gabrielle (1994), "Intermediate preferences and stable coalition structures," Journal of Mathematical Economics 23, 45-58.

Demange, Gabrielle and Roger Guesnerie (1997), "Nonemptiness of the core: low dimensional decisions spaces and one-dimensional preferences," Research in Economics 51, 7-17.

Demange, Gabrielle and Roger Guesnerie (2001), "On coalitional stability of anonymous interim mechanisms," Economic Theory 18, 367-389.

Demange, Gabrielle (2004), "On group stability in hierarchies and networks", Journal of Political Economy 112, 4.

Economic Theory, (2001) Symposium Differential information economics, 18, 2 .

Epple, Dennis and Thomas Romer (1991), "Mobility and Redistribution," Journal of Political Economy 99, 828-858.

Epple, Dennis, David Romer and Holger Sieg (2001), "Interjurisdictorial Sorting and Majority Rule: An Empirical Analysis," Econometrica 69, 1437-1466.

Farrell Joseph and Suzanne Scotchmer (1988) "Partnerships," The Quarterly Journal of Economics 103, 279-297.

Gabszewicz, Jean J. and Jean-Franois Thisse (1986), "Spatial competition and the location of firms," in Location Theory, Gabszewicz, Thisse, Fujita, and Schweizer eds Chur, Harwood Academic Publishers.

Gabszewicz, Jean J. and Jean-Franois Thisse (2002), Microeconomic theories of imperfect competition, Old problems and new perspectives, Elgar Reference collection, Cheltenham,UK. Northampton, MA, USA.

Gale David, and Lloyd S. Shapley (1962) "College Admissions and stability of marriage," The American Mathematical monthly 60, 9-15.

Grandmont, Jean-Michel (1978), "Intermediate Preferences and the Majority Rule," Econometrica 46, 317-330.

Greenberg, Joseph and B. Shitovitz (1988), " Consistent vong rules for competitive local public good economies," Journal of Economic Theory 46, 223-236.

Greenberg, Joseph and Shlomo Weber (1986), "Strong Tiebout Equilibrium under Restricted Preferences Domain", Journal of Economic Theory 38, 101117. 
Greenberg, J. and S. Weber (1994) "Stable coalition structures in consecutive games", in Frontiers in Game Theory, Binmore, K., Kirman, A. and P. Tani, eds., MIT Press, Cambridge.

Greenberg, Joseph and Shlomo Weber (1993), "Stable Coalition Structures with Unidimensional Set of Alternatives," Journal of Economic Theory 60, 62-82.

Guesnerie, Roger and Claude Oddou (1981), "Second best taxation as a game," Journal of Economic Theory 25, 1, 67-91.

Guesnerie, Roger and Claude Oddou (1987), "Increasing returns to size and their limits," Scandinavian Journal of Economics 90, 259-273.

Haeringer, Guillaume (2000), "Stable coalition structures with fixed decision schemes", UFAE and IAE Working Papers 471.00.

Hotelling, Harold (1929), "Stability in Competition", Economic Journal 39, $4-57$.

Jaramillo Fernando, Hubert Kempf and Fabien Moizeau (2004), " Inequality and growth clubs", this volume.

Jehiel, Philippe and Suzanne Scotchmer (2001), "Constitutional Rules of Exclusion in Jurisdiction Formation", Review of Economic Studies 68, 393-413.

Kalai, Ehud, Andrew Postlewaite and John Roberts (1978), "Barriers to Trade and Disadvantageous Middlemen:Nonmonotonicity of the Core," Journal of Economic Theory 19, 200-210.

Kaneko, Mamoru and Myrna Wooders (1982), "Cores of Partitioning Games," Mathematical Social Sciences 3, 313-327.

Kemeny, John G. and J. Laurie Snell (1962), Mathematical Models in the Social Sciences, Ginn, New York.

Kovalenkov Alexander and Myrna Wooders (2004), "Games as models of economies with large and small effective groups," this volume.

Rose-Ackerman Susan (1979): " Market models of local governments: exit, voting and the land market," Journal of Urban Economics 6, 319-337.

Rothschild, Michael and Joseph Stiglitz (1976), "Equilibrium in competitive Insurance Markets: An essay on the economics of imperfect information," Quarterly Journal of Economics 90, 629-649.

Shaked, Avner and John Sutton (1983), "Natural Oligopolies," Econometrica 51, 1469-1483.

Shapley, Lloyd S., (1967), "On Balanced Sets and Cores," Naval Research Logistics Quarterly 14, 453-460.

Shapley Llyod, S. (1971) "Cores of convex games," International Journal of Game Theory 11-26.

Sharkey, William W., (1982), The theory of natural monopoly, Cambridge University Press.

Tiebout, Charles (1956), "A Pure Theory of Local Expenditures," Journal of Political Economy 64, 416-424. 
Westhoff, F. (1977) "Existence of equilibria in economies with a local public good", Journal of Economic Theory 17, 84-112.

Wilson, R. (1978) "Information, efficiency, and the core of an economy" Econometrica 52, 1365-1368.

Yi, Sang-Seung (1997), "Stable coalition structures with externalities" Games and economic behavior 20, 201-237. 